$\left\{\begin{array}{l}\text { JOURNAL OF ETHNOBIOLOGY } \\ \text { AND ETHNOMEDIIINE }\end{array}\right.$

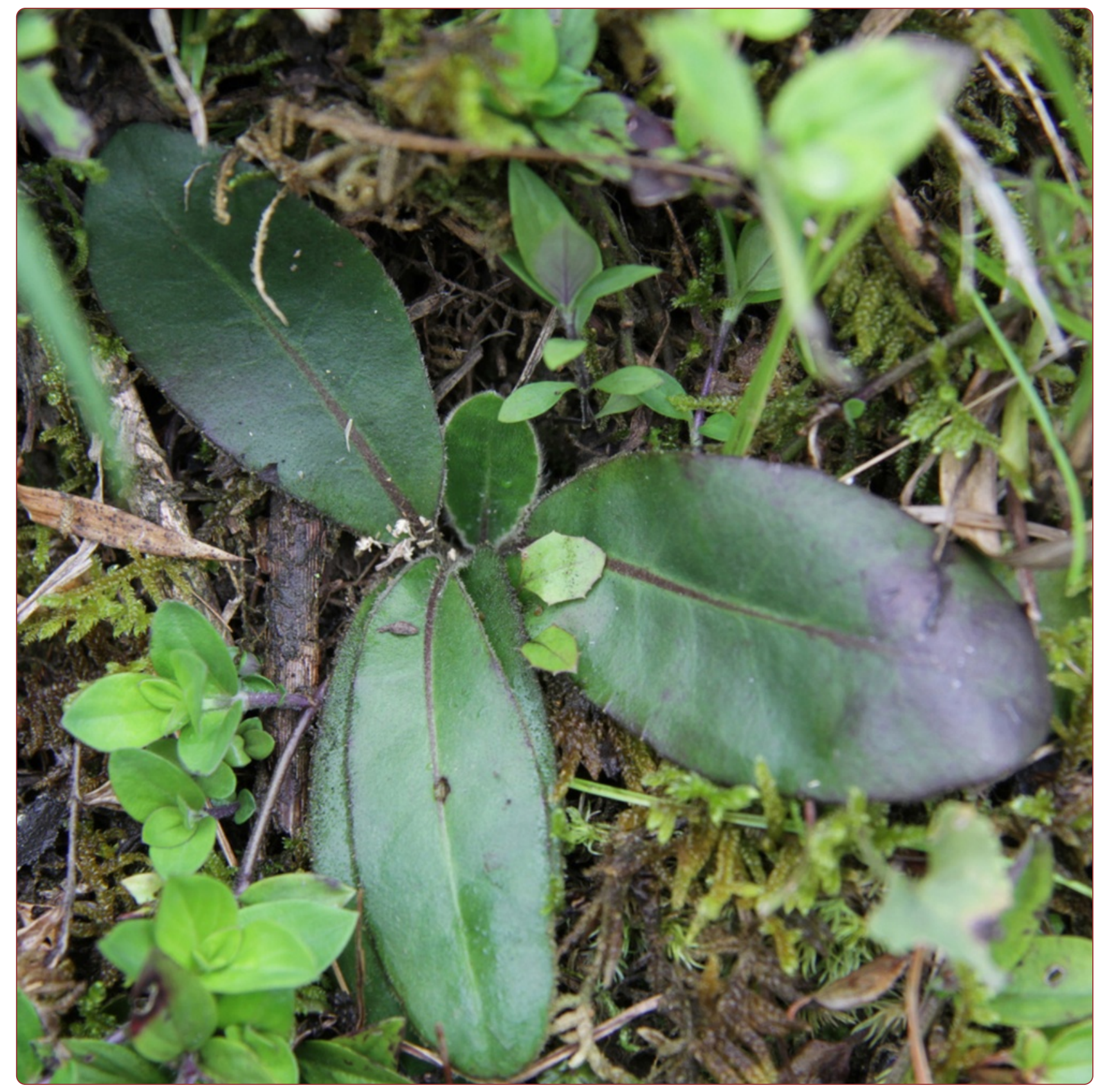

\title{
Ethnobotany of wild plants used for starting fermented beverages in Shui communities of southwest China
}

Hong et al. 


\title{
Ethnobotany of wild plants used for starting fermented beverages in Shui communities of southwest China
}

Liya Hong ${ }^{1}$, Jingxian Zhuo ${ }^{2,3}$, Qiyi Lei ${ }^{4}$, Jiangju Zhou ${ }^{4}$, Selena Ahmed ${ }^{5}$, Chaoying Wang ${ }^{6}$, Yuxiao Long ${ }^{7}$, Feifei $\mathrm{Li}^{1}$ and Chunlin Long ${ }^{1,2^{*}}$

\begin{abstract}
Background: Shui communities of southwest China have an extensive history of using wild plants as starters (Xiaoqu) to prepare fermented beverages that serve important roles in interpersonal relationships and cultural events. While the practice of using wild plants as starters for the preparation of fermented beverages was once prevalent throughout China, this tradition has seen a decline nationally since the 1930s. The traditional technique of preparing fermented beverages from wild plant starters remains well preserved in the Shui communities in southwest China and provides insight on local human-environment interactions and conservation of plant biodiversity for cultural purposes. The present study sought to examine the ethnobotany of wild plants used as starters for the preparation of fermented beverages including an inventory of plants used as a starter in liquor fermentation and associated knowledge and practices.
\end{abstract}

Methods: Field surveys were carried out that consisted of semi-structured surveys and plant species inventories. One hundred forty-nine informants in twenty Shui villages were interviewed between July 2012 and October 2014 to document knowledge associated with wild plants used as a liquor fermentation starter. The inventories involved plant voucher specimens and taxonomic identification of plant collections.

Results: A total of 103 species in 57 botanical families of wild plants were inventoried and documented that are traditionally used as starters for preparing fermented beverages by Shui communities. The majority of the species (93.2\%) have multiple uses in addition to being used as a starter with medicinal purposes being the most prevalent. Shui women are the major harvesters and users of wild plants used as starters for preparing fermented beverages and transfer knowledge orally from mother to daughter.

Conclusions: Findings from this study can serve as a basis for future investigation on fermented beverages and foods and associated knowledge and cultural practices. However, with rapid development, utilization of wild plants and the cultural systems that support them are at risk of erosion. Cultural preservation practices are necessary in Shui communities for the continued use and transmission of this ethnobiological knowledge as well as associated biodiversity.

Keywords: Fermented beverages, Biodiversity, Xiaoqu starter, Traditional ethnobotanical knowledge, Cultural preservation

\footnotetext{
*Correspondence: long@mail.kib.ac.cn

${ }^{1}$ College of Life and Environmental Sciences, Minzu University of China,

Beijing 100081, People's Republic of China

${ }^{2}$ Kunming Institute of Botany, Chinese Academy of Sciences, Kunming

650201, People's Republic of China

Full list of author information is available at the end of the article
} 


\section{Background}

Fermented food and beverages that preserve diverse, locally available resources have been consumed for centuries worldwide as notable dietary components to support household food security and overall wellbeing [1-10]. Traditionally, such products were associated with cultural identity and social aspects of communities and were most often prepared at the household-scale through the action of microorganisms and their enzymes [10,11]. Key characteristics of fermented foods and beverages are enhancements to flavor and/or appearance, preserved quality, prolonged shelf-life, reduced cooking time and prebiotic and probiotic properties that have benefits for increasing digestability and bioavailablilty of certain nutrients [12-19]. Various cultures around the world prepare and consume fermented products to enhance their basic diet including as a side dish, condiment, pickle, confection and beverage. Knowledge on the preparation and attributes of fermented foods and beverages has been transferred from generation to generation and represents traditional ethnobiological knowledge.

In many minority socio-linguistic groups throughout China, the preparation and consumption of fermented alcoholic beverages are important cultural practices that define many social interactions including rituals during courtship, engagements, weddings, hospitality, funerals, ancestor worship and other ceremonies [20]. Various socio-linguistic groups have their own characteristics of preparing and consuming fermented alcoholic beverages that contribute to cultural identity such as Mongolian koumiss, Yi's spicy liquor, Tibetan highland barley wine and Shui's Jiuqianjiu liquor.

The Shui prepare fermented alcoholic beverages known in Chinese as Jiuqianjiu liquor and in Shui as Kaojiuqian that is made from water, rice and a special starter made of wild plants known as Xiaoqu. Although the origin is not clear, Jiuqianjiu liquor is a traditional fermented beverage that has long been prepared and consumed in Shui communities as an integral part of daily life as well as for celebratory reasons including the main Shui holiday that occurs in the lunar calendar during September. The production of Jiuqianjiu liquor involves harvesting wild plants and the wild type microbe inoculation of molds, yeasts and bacteria in a rice (or other grain) substrate. The whole process of making this starter from plant collecting to material mixing, shaping, ripening, drying and storing usually takes 3 months. The Xiaoqu starter is often regarded as the most important ingredient for determining the quality of the final beverage. The production of Jiuqianjiu liquor can be compared to the Japanese process of Koji. Diverse wild plants are used as a starter for preparing fermented alcoholic beverages in Shui and other socio-linguistic groups of southwestern China. Indigenous communities in southwestern China believe that the synergy of different wild plants with microorganisms modifies the environment for the microbes by providing nutrition and inhibiting the growth of detrimental microbes and ultimately can modify the flavor and health attributes of the final fermented product.

The use of wild plants as a starter for fermented beverages has a long history in China. The earliest known recording is by Qi Min Yao Shu during the years 533-544. With technological development, pure breeding fermentation technologies that were introduced to China in the 1930s replaced the use of wild plants as a fermentation starter in most areas throughout the country. In the present era, socio-economic and political influences in China's rural areas are threatening traditional practices of preparing fermented foods and beverages along with their associated knowledge base.

Understanding the ethnobotany of wild plants used as a starter for preparing fermented beverages can serve as a basis for future studies and applications regarding fermented products. However, this information has not been documented in most communities where this practice remains prevalent. The present study tries to address this knowledge gap through an ethnobotanical investigation of wild plants used as starters for fermented beverages in indigenous Shui communities of southwest China. Specifically, our study aimed to address the following objectives in Shui communities: (1) characterize the plants used as starters to prepare fermented alcoholic beverages, (2) document associated ethnobotanical knowledge, and (3) record the processing of fermented alcoholic beverages.

\section{Materials and methods}

\section{Study area and Shui people}

Surveys were conducted in Sandu Shui Autonomous County located in the south of Guizhou Province of China $\left(25^{\circ} 30^{\prime}-25^{\circ} 10^{\prime} \mathrm{N}, 107^{\circ} 40^{\prime}-108^{\circ} 14^{\prime}\right.$ E). It is in Qiannan Prefecture, Guizhou province where the Shui population is most densely settled and where the Shui people regard it as their cultural and linguistic center [21]. This area was selected for research because it is floristically rich with a cultural practice of drawing on this biodiversity for preparation of fermented beverages. Sandu Shui Autonomous County is characterized by numerous high mountains and large and small rivers. The climate is considered as a subtropical humid monsoon type with long summers and short winters. The complexity of the terrain, topography and altitude of the county has resulted in enormous variations of the climate. The country has a total area of 2,380 square kilometers with a total of 237,588 people living in 270 villages.

With an approximate population of 406,900 , the Shui are one of the 55 officially recognized minority nationalities 
in China. Sandu Shui Autonomous County is the only county in China dominated by Shui people. The Shui people account for $65.93 \%$ of the total population of this prefecture; the remaining population consists of Han, Buyi, Miao, Yao and nine other minority socio-linguistic groups. They are distributed in Guizhou, Guangxi, Yunnan and Sichuan Provinces. The Shui language belongs to the Kam-Shui language grouping within the Sino-Tibetan language family $[21,22]$. Due to the long communication with the Han, almost all Shui now know both Shui and Chinese Mandarin languages. While the Shui people have their own written language as found in the "Shui Book", Chinese has become the written language in their daily life.
The Shui also have their own calendar that dictates their ceremonies.

These Shui villages in mountainous valleys and basins are usually located near rivers and even today display the stilted wooden house style. The Shui at the study sites live in clusters of small-scaled villages. Until some decades ago there was a custom that a Shui woman should get married to one of her father's sister's son (cross-cousin marriage). Some have suggested that cross-cousin bonds reflected an original clan-based social organization with compact communities and villages typically of a few hundred related by bloodlines [21, 23]. Historically, they were not allowed to intermarry with

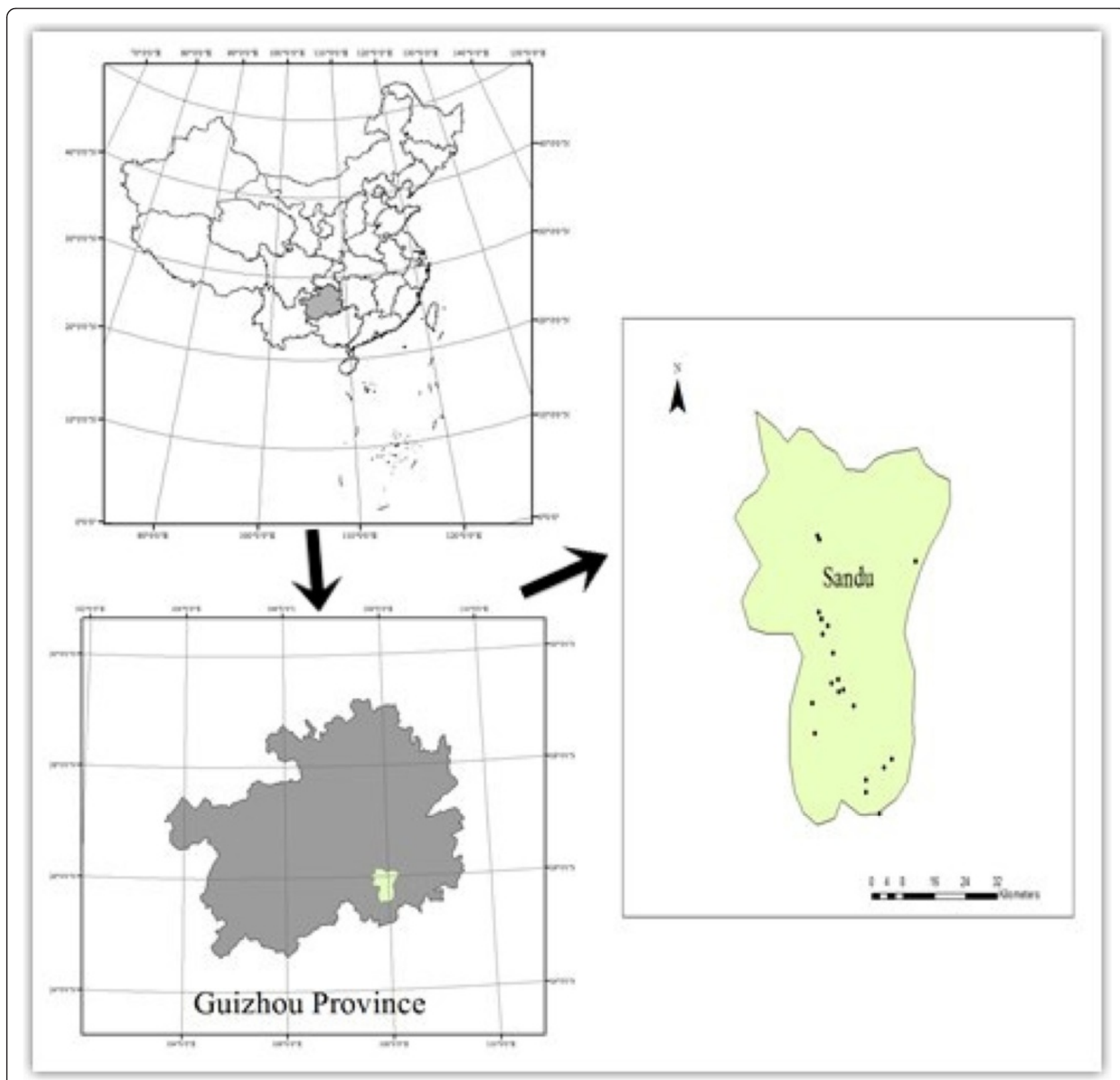

Fig. 1 Sketch map of the study area, Sandu County of Guizhou 
other nationalities. Similar to other indigenous groups in the area, the Shui follow polytheism and animism with worship of ancestors and natural objects including mountains, rocks and ancient trees. Traditional lifestyle is still common in the area and the Shui people are fond of pickles and sour soup in their daily diet. Staple food of the Shuis is rice, together with different local vegetables and meat as protein source. Traditional practices are still common in Shui communities including the production of fermented alcoholic beverages.

Field studies were carried out in 20 villages located in five townships in Sandu County including: Banlong, Bangao (Zhouqin town), Shuitiao, Dabian, Layou, Duzhai, Malian, Shuilong, Sanhe, Bamao, Banmiao, Miaoliang, Zenlei, Shuige, Bangao (Jiuqian town), Shuigen, Shuixi, Shuimei, Yanpai and Banqi villages. The villages were randomly selected from all Shui villages in Sandu County to carry out ethnobotanical investigations (Fig. 1). The majority of individuals in the villages are Shui. The population varies from 253 to 2,252 in each village. No notable geographical and environmental differences exist in the study area.

\section{Field surveys}

Field surveys were carried out between July 2012 and October 2014 using participatory rural appraisal (PRA) and ethnobotanical methods [24-27] in order to document the species, habitats and varied uses of plants used as starters for preparing fermented beverages (Fig. 2). A total of 149 informants including 32 males and $117 \mathrm{fe}$ males were interviewed (seven to ten people per village). Informants were between the ages of 23 and 84 years old. Fifty-three key informants were identified who were highly respected in their communities for their rich knowledge of plants used for starters for fermented beverages. Key informants included 38 village elders, 10 traditional brewers of fermented beverages and 5 managers of local liquor distilleries. In addition, ninety-six randomly selected households were surveyed. Permissions were provided by all participants in this study, including the Shui people.

During each visit, documented plant species were collected from different habitats around the study sites (Fig. 3). In addition, surveys documented vascular names, parts used, frequency of use and other values held by informants during interviews as well as through participant observation. Scientific name, botanical family name, growth forms, plant conservation status and other values, such as medicinal, edible, ornamental, spice, dyeing, herbal tea, fence and timber, were recorded for each plant species in Table 1. The collected ethnobotanical data were summarized using descriptive statistical analysis including frequency and their values. Frequency was used to determine the relative importance of plant species. Voucher specimens were collected, identified and deposited in the Herbarium at the College of Life and Environmental Sciences of Minzu University of China. Family assignation in this paper followed the Flora of China and TROPICOS.

\section{Results and discussion}

\section{Diversity of plants used as starters for preparing} fermented beverages

A total of 103 wild-harvested plant species were documented as starters for preparing fermented alcoholic beverages at the study sites. This includes species distributed in 88 genera and 57 families consisting of 97 species of angiosperms, 2 species of gymnosperms, and 4 species of pteridophytes. The majority of plants belonged to the Asteraceae (12 species), Rosaceae (9), Fabaceae (6), Melastomaceae (3), Moraceae (3) and Rutaceae (3). Table 1 lists

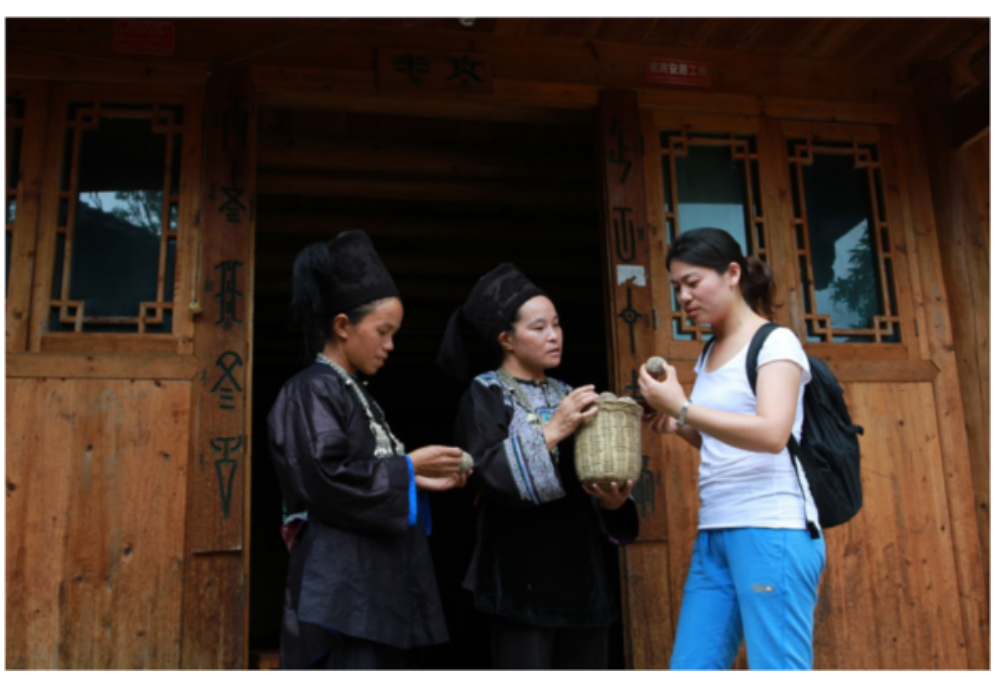

Fig. 2 Key informant interview 


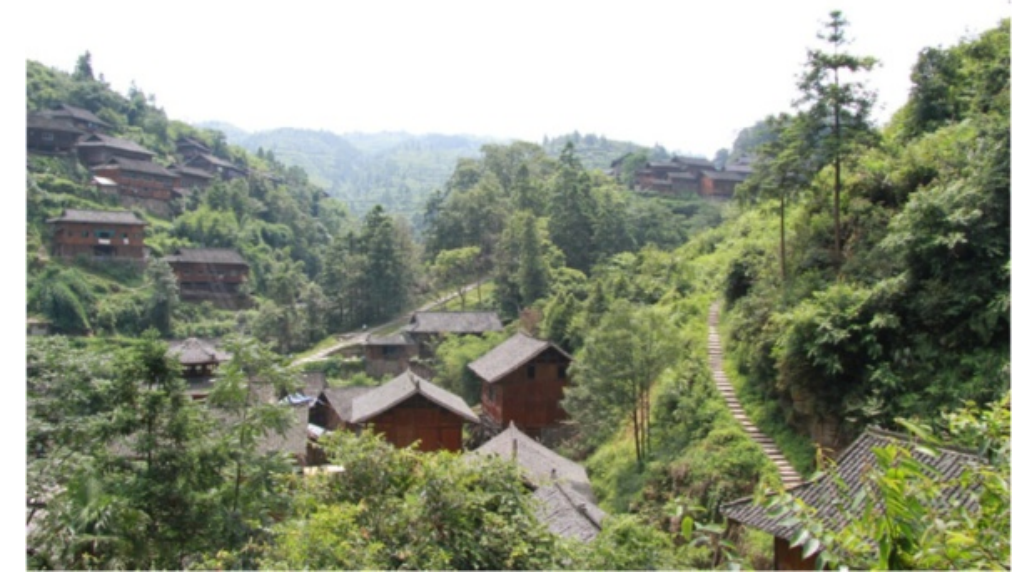

Fig. 3 A Shui village investigated

the Chinese name, Shui name, scientific name, family name, habitat, plant parts used, frequency of utilization and their values. The recorded species occur as various life forms with the majority being herbaceous species (42\%) and the remaining occurring as shrubs (32\%), trees (17\%), lianas (12\%), and epiphytes (4\%) (Fig. 4). All of the total 103 plant species were wild harvested from montane forests, wetlands, shrub lands, and wastelands. Most of the wild plant species had wide distribution in the study sites and were easily accessible.

The average number of species mentioned per informant for use as a starter for the preparation of fermented alcoholic beverages was 15 while the average number for key informants was 54 species. The most frequently mentioned plants by informants include Gerbera piloselloides (Fig. 5), Lygodium japonicum (Fig. 6), Rosa roxburghii (Fig. 7), Paederia foetida, Zanthoxylum bungeanum, Plantago depressa and Platycodon grandiflorus. Informants use varied plant parts for preparing the fermented culture including leaves, roots, barks and fruits (Fig. 8). The majority of plants are harvested for their leaves (52 species documented) followed by aerial parts (26). Informants also use whole plants (17), fruits (15) as well as other parts.

Documentation of local wild floristic diversity used by Shui communities as starters for the preparation of fermented beverages is important to support conservation and cultural revitalization efforts in the study site communities. All of the 103 plant species documented as starters are wild harvested and reflect the rich biological diversity in the study area. The greater use of herbaceous plants compared to other types of plants such as woody plant species might be due to their ease of collection, higher abundance, and high effectiveness in comparison to other life-forms. Previous ethnobotanical studies have found that weedy and herbaceous plants are often the majority of plants collected for food, medicine, and other purposes [27]. In addition, various plant parts are use for fermentation with the leaf being the most prevalent plant part used. Previous research in minority sociolinguistic communities in China has shown similar results of leaves being the most prevalent part harvested; this might be due to ease of collection and less threat presented to harvested resources as the practice of harvesting the whole plant or roots presents greater threats to survival [28-30]. In addition, the prevalence of collecting herbaceous plants versus other plant types might also be due to socio-cultural beliefs and practices of the Shui as well as environmental factors. Communities may harvest shrubs, trees, and lianas for their ability to withstand long dry seasons that result in their availability throughout the year as well as increased availability during the harvest period that wild plants are generally collected for starters. Seasons when plants bloomed and fruited, typically during the summer, are also when it is easiest to identify plants for collection. Furthermore, the summer is the best season for microbial fermentation of food and beverages because of the higher temperature promotes fermentation activity. Preparation during the summer also fits within the local Shui calendar as this is after the harvest of rice that rice is used as a raw material input for fermented beverages.

\section{Multiple uses and economic value of the wild plants}

In addition to fermentation purposes, informants use the documented wild plants for multiple other purposes. Almost all documented wild plants $(98.1 \%)$ are locally valued for their medicinal, edible, ornamental, spice, dye, herbal tea, fence and timber uses with medicinal purposes for the prevention and treatment of different health conditions being the most prevalent use (Table 2). Specifically, the most common medicinal function of 
Table 1 Plants used for liquor fermentation starter in Sandu County of Guizhou

\begin{tabular}{|c|c|c|c|c|c|c|c|c|c|}
\hline No. & Chinese name & & Shui name & Scientific name & Family name & $\begin{array}{l}\text { Life } \\
\text { form }\end{array}$ & $\begin{array}{l}\text { Parts } \\
\text { used }\end{array}$ & Frequency & Other value \\
\hline 1 & Tuniuxi & $\begin{array}{l}\text { 土 } \\
\text { 牛 } \\
\text { 膝 }\end{array}$ & $m a^{24} \operatorname{dian}^{43}$ & $\begin{array}{l}\text { Achyranthes } \\
\text { aspera } \mathrm{L} \text {. }\end{array}$ & Amaranthaceae & Herb & Leaf & $* * *$ & $\begin{array}{l}\text { Clearing away heat and } \\
\text { toxic materials; Diuretics; } \\
\text { Cold; Fever; Amygdalitis; } \\
\text { Mumps }\end{array}$ \\
\hline 2 & $\begin{array}{l}\text { Zhonghua- } \\
\text { mihoutao }\end{array}$ & $\begin{array}{l}\text { 中 } \\
\text { 华 } \\
\text { 猕 } \\
\text { 猴 } \\
\text { 桃 }\end{array}$ & $\mathrm{fan}^{43} \mathrm{ma}^{24}$ & $\begin{array}{l}\text { Actinidia } \\
\text { chinensis Planch. }\end{array}$ & Actinidiaceae & Shrub & Leaf & $* * * *$ & The fruit is edible \\
\hline 3 & Geyemihoutao & $\begin{array}{l}\text { 革 } \\
\text { 叶 } \\
\text { 猕 } \\
\text { 猴 } \\
\text { 桃 }\end{array}$ & $\mathrm{fan}^{43} \mathrm{ma}^{24}$ & $\begin{array}{l}\text { Actinidia } \\
\text { rubricaulis var. } \\
\text { coriacea (Finet \& } \\
\text { Gagnep.) C.F. } \\
\text { Liang }\end{array}$ & Actinidiaceae & Shrub & $\begin{array}{l}\text { Leaf, } \\
\text { Fruit }\end{array}$ & $* * *$ & The fruit is edible \\
\hline 4 & Longyacao & $\begin{array}{l}\text { 龙 } \\
\text { 芽 } \\
\text { 草 }\end{array}$ & $\mathrm{ga}^{31} \mathrm{jun}^{35} \mathrm{gan}^{42}$ & $\begin{array}{l}\text { Agrimonia pilosa } \\
\text { Ledeb. }\end{array}$ & Rosaceae & Herb & $\begin{array}{l}\text { Aerial } \\
\text { part }\end{array}$ & $* * * * *$ & Stopping bleed \\
\hline 5 & $\begin{array}{l}\text { Xingxiang- } \\
\text { tu'erfeng }\end{array}$ & $\begin{array}{l}\text { 杏 } \\
\text { 香 } \\
\text { 兔 } \\
\text { 儿 } \\
\text { 风 }\end{array}$ & t6yn ${ }^{35} \operatorname{lan}^{31}$ thu $^{31} \mathrm{nu}^{24} \operatorname{lun}^{42}$ & $\begin{array}{l}\text { Ainsliaea fragrans } \\
\text { Champ. ex } \\
\text { Benth. }\end{array}$ & Asteraceae & Herb & $\begin{array}{l}\text { Aerial } \\
\text { part }\end{array}$ & $* * * * *$ & $\begin{array}{l}\text { Heat-clearing and } \\
\text { detoxifying effect; } \\
\text { Clearing away heat and } \\
\text { toxic materials; Diuretics; } \\
\text { Hematemesis; Traumatic } \\
\text { injury }\end{array}$ \\
\hline 6 & Sanyemutong & $\begin{array}{l}\text { 三 } \\
\text { 叶 } \\
\text { 木 } \\
\text { 通 }\end{array}$ & $\operatorname{han}^{31}$ wa $^{24}$ mei $^{33}$ thun $^{55}$ & $\begin{array}{l}\text { Akebia trifoliata } \\
\text { (Thunb.) Koidz. }\end{array}$ & Lardizabalaceae & Liana & Leaf & $* * *$ & $\begin{array}{l}\text { Diuretics; Promoting } \\
\text { lactation; Loosing bones } \\
\text { and muscles; Removing } \\
\text { dampness; Arthralgia }\end{array}$ \\
\hline 7 & Ersexiangqing & $\begin{array}{l}\text { 至 } \\
\text { 香 } \\
\text { 青 }\end{array}$ & $\mathrm{ja}^{55} \mathrm{sa}^{43} \operatorname{lan}^{35} \mathrm{tchu}^{53}$ & $\begin{array}{l}\text { Anaphalis bicolor } \\
\text { (Franch.) Diels }\end{array}$ & Asteraceae & Shrub & $\begin{array}{l}\text { Whole } \\
\text { plant }\end{array}$ & $* *$ & $\begin{array}{l}\text { Removing dampness; } \\
\text { Relieving summer-heat; } \\
\text { Cough }\end{array}$ \\
\hline 8 & Jinxiancao & $\begin{array}{l}\text { 金 } \\
\text { 线 } \\
\text { 草 }\end{array}$ & nian ${ }^{55} \mathrm{ha}^{24} \mathrm{gan}^{43}$ & $\begin{array}{l}\text { Antenoron } \\
\text { filiforme (Thunb.) } \\
\text { Roberty \& Vautier }\end{array}$ & Polygonaceae & Herb & $\begin{array}{l}\text { Aerial } \\
\text { part }\end{array}$ & $* * * *$ & $\begin{array}{l}\text { Dispelling wind and } \\
\text { cold; Removing } \\
\text { dampness; Relieving } \\
\text { pain; Stopping bleed; } \\
\text { Eliminating stasis to } \\
\text { stop pain }\end{array}$ \\
\hline 9 & Zijinniu & $\begin{array}{l}\text { 紫 } \\
\text { 金 } \\
\text { 牛 }\end{array}$ & $\operatorname{tgin}^{24}$ nian $^{24}$ kui $^{42}$ & $\begin{array}{l}\text { Ardisia japonica } \\
\text { (Thunb.) Bl. }\end{array}$ & Myrsinaceae & Shrub & $\begin{array}{l}\text { Aerial } \\
\text { part }\end{array}$ & $* * * * *$ & $\begin{array}{l}\text { Phthisis; Hemoptysis; } \\
\text { Cough; Tracheitis; } \\
\text { Removing dampness; } \\
\text { Leukorrhea; } \\
\text { Amenorrhoea; Diuretics }\end{array}$ \\
\hline 10 & Qihao & $\begin{array}{l}\text { 奇 } \\
\text { 蒿 }\end{array}$ & $\mathrm{n}_{\mathbf{o}} \mathrm{i}^{35} \mathrm{ai}^{35}$ & $\begin{array}{l}\text { Artemisia } \\
\text { anomala S. } \\
\text { Moore }\end{array}$ & Asteraceae & Herb & $\begin{array}{l}\text { Aerial } \\
\text { part }\end{array}$ & $* * *$ & $\begin{array}{l}\text { Removing dampness; } \\
\text { Promoting blood } \\
\text { circulations; Eliminating } \\
\text { stasis to stop pain; } \\
\text { Irregular menstruation; } \\
\text { Relieving pain }\end{array}$ \\
\hline 11 & Hualianxixin & $\begin{array}{l}\text { 花 } \\
\text { 脸 } \\
\text { 细 } \\
\text { 辛 }\end{array}$ & $n u^{55} n a^{55} t^{35} n a^{24}$ & $\begin{array}{l}\text { Asarum splendens } \\
\text { (F. Maekawa) C. } \\
\text { Y. Cheng \& C. S. } \\
\text { Yang }\end{array}$ & Aristolochiaceae & Herb & $\begin{array}{l}\text { Whole } \\
\text { plant }\end{array}$ & $* * * *$ & $\begin{array}{l}\text { Dispelling wind and } \\
\text { cold; Relieving pain; } \\
\text { Warming lung for } \\
\text { dispelling cold }\end{array}$ \\
\hline 12 & Wulingxixin & $\begin{array}{l}\text { 五 } \\
\text { 岭 } \\
\text { 细 } \\
\text { 辛 }\end{array}$ & $w \partial^{33} n u^{33} t \partial^{35} n a^{24}$ & $\begin{array}{l}\text { Asarum } \\
\text { wulingense C.F. } \\
\text { Liang }\end{array}$ & Aristolochiaceae & Herb & $\begin{array}{l}\text { Whole } \\
\text { plant }\end{array}$ & $* * *$ & $\begin{array}{l}\text { Dispelling wind and } \\
\text { cold; Relieving pain; } \\
\text { Warming lung for } \\
\text { dispelling cold }\end{array}$ \\
\hline 13 & Tiejiaojue & $\begin{array}{l}\text { 铁 } \\
\text { 角 } \\
\text { 蕨 }\end{array}$ & $6 e^{24} \operatorname{pau}^{43} t^{35}$ & $\begin{array}{l}\text { Asplenium } \\
\text { trichomanes L. }\end{array}$ & Aspleniaceae & Herb & $\begin{array}{l}\text { Whole } \\
\text { plant }\end{array}$ & $* *$ & $\begin{array}{l}\text { Heat-clearing and } \\
\text { detoxifying effect; } \\
\text { Removing dampness; } \\
\text { Stopping bleed; } \\
\text { Eliminating stasis to }\end{array}$ \\
\hline
\end{tabular}


Table 1 Plants used for liquor fermentation starter in Sandu County of Guizhou (Continued)

\begin{tabular}{|c|c|c|c|c|c|c|c|c|}
\hline & & & & & & & & $\begin{array}{l}\text { stop pain; Dysentery; } \\
\text { Leukorrhea; Irregular } \\
\text { menstruation; Backache }\end{array}$ \\
\hline 14 & Ziwan & $\begin{array}{l}\text { 紫 } t_{6 i e^{35}} y_{u} e^{55} \\
\text { 苑 }\end{array}$ & $\begin{array}{l}\text { Aster ageratoides } \\
\text { Turcz. }\end{array}$ & Asteraceae & Herb & $\begin{array}{l}\text { Aerial } \\
\text { part }\end{array}$ & $* * * *$ & $\begin{array}{l}\text { Cold; Cough; Asthma; } \\
\text { Consumptive disease; } \\
\text { Blood vomitting }\end{array}$ \\
\hline 15 & Sanmaiziwan & $\begin{array}{l}\text { 戛 } \\
\text { 紫 } \\
\text { 苑 }\end{array}$ & $\begin{array}{l}\text { Aster tataricus } \mathrm{L} . \\
\mathrm{f} .\end{array}$ & Asteraceae & Herb & $\begin{array}{l}\text { Aerial } \\
\text { part }\end{array}$ & $* * *$ & $\begin{array}{l}\text { Cold; Cough; Asthma; } \\
\text { Consumptive disease; } \\
\text { Blood vomitting }\end{array}$ \\
\hline 16 & Xianghua & $\begin{array}{l}\text { 香 } \operatorname{lan}^{24} h_{u} a^{255} \\
\text { 华 }\end{array}$ & $\begin{array}{l}\text { Betula insignis } \\
\text { Franch. }\end{array}$ & Betulaceae & Tree & Leaf & $* * * * *$ & $\begin{array}{l}\text { Cough; Asthma; } \\
\text { Eliminating phlegm }\end{array}$ \\
\hline 17 & Baiji & 帛 $\mathrm{ja}^{43} \mathrm{pau}^{43}$ & $\begin{array}{l}\text { Bletilla striata } \\
\text { (Thunb.) Rchb. f. }\end{array}$ & Orchidaceae & Herb & Root & $* * * * *$ & $\begin{array}{l}\text { Stopping bleed; Anti- } \\
\text { swelling agent; Promot- } \\
\text { ing tissue regeneration }\end{array}$ \\
\hline 18 & Laijiangteng & $\begin{array}{l}\text { 来 } \mathrm{dan}{ }^{42} \mathrm{kui}^{33} \mathrm{jau}^{43} \\
\text { 江 } \\
\text { 藤 }\end{array}$ & $\begin{array}{l}\text { Brandisia hancei } \\
\text { Hook. f. }\end{array}$ & Scrophulariaceae & Shrub & $\begin{array}{l}\text { Stem, } \\
\text { Leaf }\end{array}$ & $* * * * *$ & $\begin{array}{l}\text { Clearing away heat and } \\
\text { toxic materials; Bone } \\
\text { fracture; Hepatitis }\end{array}$ \\
\hline 19 & Jinqianbao & $\begin{array}{l}\text { 金 } \operatorname{nian}^{24} 6 \mathrm{ie}^{33} \mathrm{me}^{43} \\
\text { 钱 } \\
\text { 豹 }\end{array}$ & $\begin{array}{l}\text { Campanumoea } \\
\text { javanica Bl. }\end{array}$ & Campanulaceae & Liana & $\begin{array}{l}\text { Aerial } \\
\text { part }\end{array}$ & $* * *$ & $\begin{array}{l}\text { Warming spleen and } \\
\text { stomach for dispelling } \\
\text { cold; Moistening lung to } \\
\text { arrest cough; Inducing } \\
\text { saliva and slakes thirst; } \\
\text { Spleen tonic }\end{array}$ \\
\hline 20 & Yanguantoucao & $\begin{array}{l}\text { 烟 } \mathrm{je}^{31} \operatorname{tau}^{24} \mathrm{ku}^{33} \mathrm{kan}^{43} \\
\text { 管 } \\
\text { 头 } \\
\text { 草 }\end{array}$ & $\begin{array}{l}\text { Carpesium } \\
\text { cernuum L. }\end{array}$ & Asteraceae & Herb & $\begin{array}{l}\text { Aerial } \\
\text { part }\end{array}$ & $* * * *$ & $\begin{array}{l}\text { Clearing away heat and } \\
\text { toxic materials; Anti- } \\
\text { swelling agent; Anti- } \\
\text { inflammation; Relieving } \\
\text { pain; Cold; Fever; Tooth- } \\
\text { ache; Dysentery; } \\
\text { Diuretics }\end{array}$ \\
\hline 21 & Diaodenghua & $\begin{array}{l}\text { 吊 } \mathrm{tiau}^{35} \mathrm{phau}^{33} \mathrm{nu}^{255} \\
\text { 灯 } \\
\text { 花 }\end{array}$ & $\begin{array}{l}\text { Ceropegia } \\
\text { trichantha Hemsl. }\end{array}$ & Asclepiadaceae & Liana & $\begin{array}{l}\text { Whole } \\
\text { plant }\end{array}$ & $* *$ & $\begin{array}{l}\text { Clearing away heat and } \\
\text { toxic materials; Bone } \\
\text { fracture; Hepatitis; } \\
\text { Ornamental }\end{array}$ \\
\hline 22 & Rougui & $\begin{array}{l}\text { 肉 } \mathrm{nai}^{55} \mathrm{kui}^{55} \\
\text { 桂 }\end{array}$ & $\begin{array}{l}\text { Cinnamomum } \\
\text { cassia (L.) D. Don }\end{array}$ & Lauraceae & Tree & Bark & $* * * * *$ & $\begin{array}{l}\text { Relieving pain; Waist } \\
\text { and knee pain; } \\
\text { Stomachache; } \\
\text { Dyspepsia; Amenorrhea; } \\
\text { Spice }\end{array}$ \\
\hline 23 & Ganju & $\begin{array}{l}\text { 柑 } k a: n^{55} \\
\text { 橘 }\end{array}$ & $\begin{array}{l}\text { Citrus reticulata } \\
\text { Blanco }\end{array}$ & Rutaceae & Tree & Leaf & $* * * * *$ & $\begin{array}{l}\text { Regulating qi-flowing } \\
\text { for eliminating phlegm; } \\
\text { Promoting blood circu- } \\
\text { lations; Anti-swelling } \\
\text { agent; The fruit is edible }\end{array}$ \\
\hline 24 & Dangshen & $\begin{array}{l}\text { 党 } \tan ^{24} \operatorname{sen}^{24} \\
\text { 参 }\end{array}$ & $\begin{array}{l}\text { Codonopsis } \\
\text { pilosula (Franch.) } \\
\text { Nannf. }\end{array}$ & Campanulaceae & Liana & $\begin{array}{l}\text { Aerial } \\
\text { part }\end{array}$ & $* * * * *$ & $\begin{array}{l}\text { Invigorating the blood; } \\
\text { Spleen and stomach } \\
\text { tonic; Weakness; The } \\
\text { root is cooking for } \\
\text { edible }\end{array}$ \\
\hline 25 & Fanbaocao & $\begin{array}{l}\text { 饭 } \mathrm{ma}^{33} \mathrm{kai}^{43} \mathrm{kei}^{33} \\
\text { 包 } \\
\text { 草 }\end{array}$ & $\begin{array}{l}\text { Commelina } \\
\text { bengalensis } \mathrm{L} \text {. }\end{array}$ & Commelinaceae & Herb & $\begin{array}{l}\text { Aerial } \\
\text { part }\end{array}$ & $* * *$ & $\begin{array}{l}\text { Clearing away heat and } \\
\text { toxic materials; Diuretics; } \\
\text { Anti-swelling agent; The } \\
\text { leaves and stems are } \\
\text { cooking as vegetables }\end{array}$ \\
\hline 26 & $\begin{array}{l}\text { Xianggang- } \\
\text { sizhaohua }\end{array}$ & 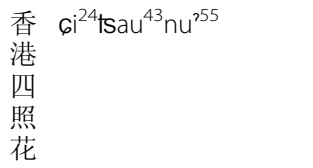 & $\begin{array}{l}\text { Cornus } \\
\text { hongkongensis } \\
\text { Hemsl. }\end{array}$ & Cornaceae & Tree & $\begin{array}{l}\text { Leaf, } \\
\text { Fruit }\end{array}$ & $* *$ & $\begin{array}{l}\text { Ornamental, The fruit is } \\
\text { edible }\end{array}$ \\
\hline 27 & Shamu & $\begin{array}{l}\text { 杉 } \mathrm{mei}^{35} \mathrm{fa}: \mathrm{i}^{24} \\
\text { 木 }\end{array}$ & & Taxodiaceae & Tree & $\begin{array}{l}\text { Young } \\
\text { leaf }\end{array}$ & $* * *$ & Construction \\
\hline
\end{tabular}


Table 1 Plants used for liquor fermentation starter in Sandu County of Guizhou (Continued)

\begin{tabular}{|c|c|c|c|c|c|c|c|c|c|}
\hline & & & & $\begin{array}{l}\text { Cunninghamia } \\
\text { lanceolata } \\
\text { (Lamb.) Hook. }\end{array}$ & & & & & \\
\hline 28 & Qinggang & $\begin{array}{l}\text { 青 } \\
\text { 冈 }\end{array}$ & mei ${ }^{35} \mathrm{khei}^{24}$ & $\begin{array}{l}\text { Cyclobalanopsis } \\
\text { glauca (Thunb.) } \\
\text { Oerst. }\end{array}$ & Fagaceae & Tree & $\begin{array}{l}\text { Fruits, } \\
\text { Barks }\end{array}$ & $* * * *$ & $\begin{array}{l}\text { Clearing away heat and } \\
\text { toxic materials }\end{array}$ \\
\hline 29 & Yuyancao & $\begin{array}{l}\text { 鱼 } \\
\text { 眼 } \\
\text { 草 }\end{array}$ & $\mathrm{mei}^{35} \mathrm{la}^{31} \mathrm{kan}^{43}$ & $\begin{array}{l}\text { Dichrocephala } \\
\text { integrifolia (L. f.) } \\
\text { Kuntze }\end{array}$ & Asteraceae & Herb & Leaf & $* *$ & $\begin{array}{l}\text { Clearing away heat and } \\
\text { toxic materials; } \\
\text { Rremoving dampness }\end{array}$ \\
\hline 30 & Yeshi & $\begin{array}{l}\text { 野 } \\
\text { 柿 }\end{array}$ & $\mathrm{mei}^{35}$ & $\begin{array}{l}\text { Diospyros kaki } \\
\text { var. silvestris } \\
\text { Makino }\end{array}$ & Ebenaceae & Tree & Leaf & $* * *$ & The fruit is edible \\
\hline 31 & Hutuizi & $\begin{array}{l}\text { 胡 } \\
\text { 颓 } \\
\text { 子 }\end{array}$ & nuei ${ }^{35} \operatorname{liu}^{255} \mathrm{la}^{33}$ & $\begin{array}{l}\text { Elaeagnus } \\
\text { pungens Thunb. }\end{array}$ & Elaeagnaceae & Shrub & $\begin{array}{l}\text { Leaf, } \\
\text { Fruit }\end{array}$ & $* * * * *$ & $\begin{array}{l}\text { Dispelling wind and } \\
\text { cold; Removing } \\
\text { dampness; Removing } \\
\text { blood stasis; Stopping } \\
\text { bleed; Traumatic injury }\end{array}$ \\
\hline 32 & Xiangru & $\begin{array}{l}\text { 香 } \\
\text { 蔏 }\end{array}$ & $\operatorname{lan}^{35} z u^{43}$ & $\begin{array}{l}\text { Elsholtzia ciliata } \\
\text { (Thunb.) Hyl. }\end{array}$ & Lamiaceae & Herb & $\begin{array}{l}\text { Stem, } \\
\text { Leaf }\end{array}$ & $* * *$ & $\begin{array}{l}\text { Relieving exterior and } \\
\text { sweating; Removing } \\
\text { dampness; Warming } \\
\text { spleen and stomach for } \\
\text { dispelling cold; } \\
\text { Diuretics; Anti-swelling } \\
\text { agent }\end{array}$ \\
\hline 33 & Jianyerong & $\begin{array}{l}\text { 尖 } \\
\text { 叶 } \\
\text { 榕 }\end{array}$ & $6 i^{43} w^{24} z_{u n}{ }^{24}$ & $\begin{array}{l}\text { Ficus henryi } \\
\text { Warb. }\end{array}$ & Moraceae & Tree & $\begin{array}{l}\text { Leaf, } \\
\text { Fruit }\end{array}$ & $* * * *$ & $\begin{array}{l}\text { Cough; Toothache; } \\
\text { Removing dampness; } \\
\text { The fruit is edible }\end{array}$ \\
\hline 34 & Bili & $\begin{array}{l}\text { 薜 } \\
\text { 荔 }\end{array}$ & cue $e^{35} \mid i^{43}$ & Ficus pumila L. & Moraceae & Shrub & Leaf & $* * * * *$ & $\begin{array}{l}\text { Clearing away heat and } \\
\text { toxic materials; Expelling } \\
\text { damp; Diuretics; The } \\
\text { achene is washed for } \\
\text { cooking bean jelly }\end{array}$ \\
\hline 35 & Diguo & $\begin{array}{l}\text { 地 } \\
\text { 果 }\end{array}$ & $\operatorname{lan}^{43} \operatorname{an}^{35}$ & $\begin{array}{l}\text { Ficus tikoua } \\
\text { Bureau }\end{array}$ & Moraceae & Herb & $\begin{array}{l}\text { Stem, } \\
\text { Leaf, } \\
\text { Fruit }\end{array}$ & $* * *$ & $\begin{array}{l}\text { Heat-clearing and } \\
\text { detoxifying effect; } \\
\text { Removing dampness; } \\
\text { Promoting blood } \\
\text { circulations; Clearing } \\
\text { away heat and toxic } \\
\text { materials; The fruit is } \\
\text { edible }\end{array}$ \\
\hline 36 & Qianjinba & $\begin{array}{l}\text { 千 } \\
\text { 斤 } \\
\text { 拔 }\end{array}$ & sie $^{35}$ tcie $^{43}$ jun $^{33}$ & $\begin{array}{l}\text { Flemingia } \\
\text { prostrata Roxb. }\end{array}$ & Fabaceae & Shrub & Leaf & $* * * *$ & $\begin{array}{l}\text { Dispelling wind and } \\
\text { cold; Removing } \\
\text { dampness; Removing } \\
\text { blood stasis; Clearing } \\
\text { away heat and toxic } \\
\text { materials }\end{array}$ \\
\hline 37 & Zhizi & $\frac{\text { 槬 }}{\text { 子 }}$ & $m e i^{35} \mid \partial^{43}$ & $\begin{array}{l}\text { Gardenia } \\
\text { jasminoides J. } \\
\text { Ellis }\end{array}$ & Rubiaceae & Shrub & $\begin{array}{l}\text { Leaf, } \\
\text { Fruit }\end{array}$ & $* * * * *$ & $\begin{array}{l}\text { Heat-clearing and } \\
\text { detoxifying effect; } \\
\text { Purging intense heat; } \\
\text { Herbal tea; Ornamental }\end{array}$ \\
\hline 38 & Weiyebaizhu & $\begin{array}{l}\text { 尾 } \\
\text { 叶 } \\
\text { 白 } \\
\text { 珠 }\end{array}$ & hen ${ }^{55} \mathrm{wa}^{24} \mathrm{pa}^{33} \mid \mathrm{ei}^{43}$ & $\begin{array}{l}\text { Gaultheria } \\
\text { griffithiana Wight }\end{array}$ & Ericaceae & Shrub & Lef & $* * *$ & $\begin{array}{l}\text { Removing dampness; } \\
\text { Ornamental }\end{array}$ \\
\hline 39 & Baiguobaizhu & $\begin{array}{l}\text { 白 } \\
\text { 果 } \\
\text { 白 } \\
\text { 珠 }\end{array}$ & $p a^{33}\left|\mathrm{ei}^{43} \mathrm{pa}^{33}\right| \mathrm{ei}^{43}$ & $\begin{array}{l}\text { Gaultheria } \\
\text { leucocarpa Bl. }\end{array}$ & Ericaceae & Shrub & Leaf & $* * *$ & $\begin{array}{l}\text { Removing dampness; } \\
\text { Ornamental }\end{array}$ \\
\hline 40 & Maodadingcao & $\begin{array}{l}\text { 毛 } \\
\text { 大 } \\
\text { 丁 } \\
\text { 草 }\end{array}$ & $\mathrm{n}_{\mathbf{o}} \mathrm{i}^{55} \mathrm{mo}^{33} \quad i \mathrm{e}^{43}$ & $\begin{array}{l}\text { Gerbera } \\
\text { piloselloides (L.) } \\
\text { Cass. }\end{array}$ & Asteraceae & Herb & $\begin{array}{l}\text { Whole } \\
\text { plant }\end{array}$ & $* * * * *$ & $\begin{array}{l}\text { Clearing internal heat; } \\
\text { Anti-inflammatory ef- } \\
\text { fects; Cold; Fever; Post- } \\
\text { partum dysphoria }\end{array}$ \\
\hline
\end{tabular}


Table 1 Plants used for liquor fermentation starter in Sandu County of Guizhou (Continued)

\begin{tabular}{|c|c|c|c|c|c|c|c|c|}
\hline 41 & Dadingcao & $\begin{array}{l}\text { 大 } \mathrm{mo}^{33} \mathrm{Gie}^{43} \\
\text { 丁 } \\
\text { 草 }\end{array}$ & $\begin{array}{l}\text { Gerbera anandria } \\
\text { (L.) Sch.-Bip. }\end{array}$ & Asteraceae & Herb & $\begin{array}{l}\text { Whole } \\
\text { plant }\end{array}$ & $* * * *$ & $\begin{array}{l}\text { Heat-clearing and } \\
\text { detoxifying effect; } \\
\text { Removing dampness; } \\
\text { Anti-swelling agent; } \\
\text { Bleeding }\end{array}$ \\
\hline 42 & Lubianqing & $\begin{array}{l}\text { 路 } \mathrm{t}_{6 i a^{35} \mathrm{khun}^{43} \mathrm{jy}{ }^{43}} \text { 边 } \\
\text { 青 }\end{array}$ & $\begin{array}{l}\text { Geum aleppicum } \\
\text { Jacq. }\end{array}$ & Rosaceae & Herb & $\begin{array}{l}\text { Aerial } \\
\text { part }\end{array}$ & $* * * * *$ & $\begin{array}{l}\text { Irregular menstruation; } \\
\text { Dispelling wind and } \\
\text { cold; Removing } \\
\text { dampness; Relieving } \\
\text { pain }\end{array}$ \\
\hline 43 & Suanpanzi & $\begin{array}{l}\text { 算 } m e i^{24}\left|a^{33}\right| i^{43} \\
\text { 盘 } \\
\text { 子 }\end{array}$ & $\begin{array}{l}\text { Glochidion } \\
\text { puberum (L.) } \\
\text { Hutch. }\end{array}$ & Euphorbiaceae & Shrub & $\begin{array}{l}\text { Stem, } \\
\text { Leaf, } \\
\text { Fruit }\end{array}$ & $* * * * *$ & $\begin{array}{l}\text { Heat-clearing and } \\
\text { detoxifying effect; } \\
\text { Removing dampness; } \\
\text { Dispelling wind and } \\
\text { cold; Loosing bones and } \\
\text { muscles; Leukorrhea; } \\
\text { Dysmenorrhea }\end{array}$ \\
\hline 44 & Shanxiaoju & $\begin{array}{l}\text { 山 } n u^{55} t^{33} t_{6 i u^{55}} \\
\text { 小 } \\
\text { 橘 }\end{array}$ & $\begin{array}{l}\text { Glycosmis } \\
\text { pentaphylla } \\
\text { (Retz.) DC. }\end{array}$ & Rutaceae & Tree & Leaf & $* * * *$ & $\begin{array}{l}\text { Eliminating stasis to } \\
\text { stop pain; Anti-swelling } \\
\text { agent; The fruit is edible }\end{array}$ \\
\hline 45 & Nuomituan & $\begin{array}{l}\text { 糯 } \mathrm{nu}^{55} \mathrm{man}^{33} \mathrm{kan}^{43} \\
\text { 米 } \\
\text { 团 }\end{array}$ & $\begin{array}{l}\text { Gonostegia hirta } \\
\text { (Bl. ex Hassk.) } \\
\text { Miq. }\end{array}$ & Urticaceae & Herb & $\begin{array}{l}\text { Whole } \\
\text { plant }\end{array}$ & $* * * * *$ & $\begin{array}{l}\text { Spleen tonic; Digestion; } \\
\text { Heat-clearing and de- } \\
\text { toxifying effect; Remov- } \\
\text { ing dampness; Anti- } \\
\text { swelling agent }\end{array}$ \\
\hline 46 & Xiao'erxiancao & $\begin{array}{l}\text { 小 }{ }^{3}{ }^{33} \mathrm{ka}^{33} \mathrm{Gie}^{43} \mathrm{kan}^{43} \\
\text { 仙 } \\
\text { 草 }\end{array}$ & $\begin{array}{l}\text { Haloragis } \\
\text { micrantha Thunb. }\end{array}$ & Haloragidaceae & Herb & $\begin{array}{l}\text { Whole } \\
\text { plant }\end{array}$ & $* * *$ & $\begin{array}{l}\text { Diuretics; Expelling } \\
\text { damp; Clearing away } \\
\text { heat and toxic materials; } \\
\text { Antidysmenorrhea; } \\
\text { Promoting blood } \\
\text { circulations }\end{array}$ \\
\hline 47 & Changchunteng & $\begin{array}{l}\text { 常 } \mathrm{ma}^{43} \operatorname{lian} \mathrm{n}^{35} \mathrm{man}^{33} \\
\text { 春 } \\
\text { 藤 }\end{array}$ & $\begin{array}{l}\text { Hedera nepalensis } \\
\text { K. Koch }\end{array}$ & Araliaceae & Shrub & $\begin{array}{l}\text { Whole } \\
\text { plant }\end{array}$ & * & $\begin{array}{l}\text { Dispelling wind and } \\
\text { cold; Removing } \\
\text { dampness; Promoting } \\
\text { blood circulations; Anti- } \\
\text { swelling agent; } \\
\text { Ornamental }\end{array}$ \\
\hline 48 & Ercao & $\begin{array}{l}\text { 耳 } \operatorname{khan}^{43} \operatorname{kan}^{43} \\
\text { 草 }\end{array}$ & $\begin{array}{l}\text { Hedyotis } \\
\text { auricularia L. }\end{array}$ & Rubiaceae & Tree & Leaf & $* * *$ & $\begin{array}{l}\text { Heat-clearing and } \\
\text { detoxifying effect; } \\
\text { Removing dampness; } \\
\text { Anti-swelling agent }\end{array}$ \\
\hline 49 & Zhiju & $\begin{array}{l}\text { 枳 } \operatorname{ciu}^{43} \\
\text { 椇 }\end{array}$ & $\begin{array}{l}\text { Hovenia acerba } \\
\text { Lindl. }\end{array}$ & Rhamnaceae & Tree & $\begin{array}{l}\text { Leaf, } \\
\text { Fruit }\end{array}$ & $* * * *$ & $\begin{array}{l}\text { Promoting blood } \\
\text { circulations; Eliminating } \\
\text { stasis to stop pain; } \\
\text { Clearing heat and } \\
\text { expelling damp; } \\
\text { Asthma; Ornamental; } \\
\text { Fence }\end{array}$ \\
\hline 50 & $\begin{array}{l}\text { Kuanluanye- } \\
\text { changbing- } \\
\text { shanmahuang }\end{array}$ & $\begin{array}{l}\text { 宽 } \mathrm{si}^{33} \mathrm{mi}^{43} \\
\text { 卯 } \\
\text { 叶 } \\
\text { 长 } \\
\text { 柄 } \\
\text { 山 } \\
\text { 蚂 } \\
\text { 蝗 }\end{array}$ & $\begin{array}{l}\text { Hylodesmum } \\
\text { podocarpum } \\
\text { subsp. fallax } \\
\text { (Schindl.) H. } \\
\text { Ohashi \& R.R. Mill }\end{array}$ & Fabaceae & Herb & Leaf & $* * *$ & $\begin{array}{l}\text { Dispelling wind and } \\
\text { cold; Loosing bones and } \\
\text { muscles; Clearing away } \\
\text { heat and toxic materials; } \\
\text { Anti-swelling agent; } \\
\text { Traumatic injury; Remov- } \\
\text { ing dampness; Backache }\end{array}$ \\
\hline 51 & $\begin{array}{l}\text { Jianyechangbing- } \\
\text { shanmahuang }\end{array}$ & $\begin{array}{l}\text { 尖 } \mathrm{si}^{33} \mathrm{mi}^{43} \\
\text { 叶 } \\
\text { 长 } \\
\text { 柄 } \\
\text { 山 } \\
\text { 蚂 } \\
\text { 蝗 }\end{array}$ & $\begin{array}{l}\text { Hylodesmum } \\
\text { podocarpum } \\
\text { subsp. } \\
\text { oxyphyllum (DC.) } \\
\text { H. Ohashi \& R.R. } \\
\text { Mill }\end{array}$ & Fabaceae & Herb & Leaf & $* * *$ & $\begin{array}{l}\text { Dispelling wind and } \\
\text { cold; Loosing bones and } \\
\text { muscles; Clearing away } \\
\text { heat and toxic materials; } \\
\text { Anti-swelling agent; } \\
\text { Traumatic injury; Remov- } \\
\text { ing dampness }\end{array}$ \\
\hline
\end{tabular}


Table 1 Plants used for liquor fermentation starter in Sandu County of Guizhou (Continued)

\begin{tabular}{|c|c|c|c|c|c|c|c|c|c|}
\hline 52 & Tianjihuang & $\begin{array}{l}\text { 田 } \\
\text { 基 } \\
\text { 黄 }\end{array}$ & khui $^{33} \operatorname{wan}^{33} \operatorname{tin}^{43}$ & $\begin{array}{l}\text { Hypericum } \\
\text { japonicum } \\
\text { Thunb. }\end{array}$ & Clusiaceae & Herb & $\begin{array}{l}\text { Aerial } \\
\text { part }\end{array}$ & $* * * *$ & $\begin{array}{l}\text { Heat-clearing and } \\
\text { detoxifying effect; } \\
\text { Removing dampness; } \\
\text { Promoting blood } \\
\text { circulations; Anti- } \\
\text { swelling agent }\end{array}$ \\
\hline 53 & Yuanbaocao & $\begin{array}{l}\text { 元 } \\
\text { 宝 } \\
\text { 草 }\end{array}$ & juan $^{24} \operatorname{pin}^{33} \operatorname{kan}^{43}$ & $\begin{array}{l}\text { Hypericum } \\
\text { sampsonii Hance }\end{array}$ & Clusiaceae & Herb & $\begin{array}{l}\text { Whole } \\
\text { plant }\end{array}$ & $* * *$ & $\begin{array}{l}\text { Clearing away heat and } \\
\text { toxic materials; } \\
\text { Restoring menstrual } \\
\text { flow; Loosing bones and } \\
\text { muscles; Stopping } \\
\text { bleed; Fever; Dysentery; } \\
\text { Irregular } \\
\text { menstruation,Leukorrhea }\end{array}$ \\
\hline 54 & Baimao & $\begin{array}{l}\text { 白 } \\
\text { 茅 }\end{array}$ & $\mathrm{hai}^{33} \mathrm{pa}^{43}$ & $\begin{array}{l}\text { Imperata } \\
\text { cylindrica (L.) P. } \\
\text { Beauv. }\end{array}$ & Poaceae & herb & $\begin{array}{l}\text { Root, } \\
\text { Stem }\end{array}$ & $* * * * *$ & $\begin{array}{l}\text { Stopping bleed; Heat- } \\
\text { clearing and detoxifying } \\
\text { effect; Diuretics }\end{array}$ \\
\hline 55 & Jianyemulan & $\begin{array}{l}\text { 尖 } \\
\text { 叶 } \\
\text { 木 } \\
\text { 蓝 }\end{array}$ & $w^{24} \operatorname{cie}^{43}$ mei $^{35}$ tchu $^{43}$ & $\begin{array}{l}\text { Indigofera } \\
\text { zollingeriana Miq. }\end{array}$ & Fabaceae & Shrub & $\begin{array}{l}\text { Young } \\
\text { stem, } \\
\text { Leaf }\end{array}$ & $* * *$ & $\begin{array}{l}\text { Clearing away heat and } \\
\text { toxic materials; } \\
\text { Removing blood stasis; } \\
\text { Stopping bleed }\end{array}$ \\
\hline 56 & Yang'erju & $\begin{array}{l}\text { 羊 } \\
\text { 耳 } \\
\text { 菊 }\end{array}$ & $\mathrm{ma}^{35} \mathrm{hai}^{55}$ & $\begin{array}{l}\text { Inula cappa } \\
\text { (Buch.-Ham. ex } \\
\text { D. Don) DC. }\end{array}$ & Asteraceae & Shrub & $\begin{array}{l}\text { Aerial } \\
\text { part }\end{array}$ & $* * * *$ & $\begin{array}{l}\text { Dispelling wind and } \\
\text { cold; Anti-swelling } \\
\text { agent; Relieving pain }\end{array}$ \\
\hline 57 & Yuanwei & 苛 & $w^{24}{ }^{24} u^{43}$ & $\begin{array}{l}\text { Iris tectorum } \\
\text { Maxim. }\end{array}$ & Iridaceae & Herb & $\begin{array}{l}\text { Root, } \\
\text { Stem }\end{array}$ & $* * * *$ & $\begin{array}{l}\text { Promoting blood } \\
\text { circulations; Removing } \\
\text { blood stasis; Dispelling } \\
\text { wind and cold; } \\
\text { Removing dampness; } \\
\text { Clearing away heat and } \\
\text { toxic materials; } \\
\text { Digestion; Ornamental }\end{array}$ \\
\hline 58 & Nanwuweizi & $\begin{array}{l}\text { 南 } \\
\text { 五 } \\
\text { 味 } \\
\text { 子 }\end{array}$ & nai ${ }^{43}$ wo $^{24}$ wei $\left.^{24}\right|^{43}$ & $\begin{array}{l}\text { Kadsura } \\
\text { longipedunculata } \\
\text { Finet \& Gagnep. }\end{array}$ & Schisandraceae & Liana & $\begin{array}{l}\text { Whole } \\
\text { plant }\end{array}$ & $* * * *$ & $\begin{array}{l}\text { Astringent; Inducing } \\
\text { saliva and slakes thirst; } \\
\text { Notifying kidney and } \\
\text { spleen; The fruit is } \\
\text { edible }\end{array}$ \\
\hline 59 & Huorongcao & $\begin{array}{l}\text { 火 } \\
\text { 线 } \\
\text { 草 }\end{array}$ & $j y^{33} \mathrm{mau}^{43} \mathrm{kan}^{43}$ & $\begin{array}{l}\text { Leontopodium } \\
\text { leontopodioides } \\
\text { (Willd. ) Beauv. }\end{array}$ & Asteraceae & Herb & $\begin{array}{l}\text { Whole } \\
\text { plant }\end{array}$ & $* * *$ & $\begin{array}{l}\text { Clearing away heat and } \\
\text { toxic materials }\end{array}$ \\
\hline 60 & Yimucao & $\begin{array}{l}\text { 益 } \\
\text { 母 } \\
\text { 草 }\end{array}$ & $\mathrm{mai}^{33} \mathrm{kan}^{43}$ & $\begin{array}{l}\text { Leonurus } \\
\text { japonicus Houtt. }\end{array}$ & Lamiaceae & Herb & $\begin{array}{l}\text { Young } \\
\text { stem, } \\
\text { Leaf }\end{array}$ & $* * * * *$ & $\begin{array}{l}\text { Promoting blood } \\
\text { circulations; Removing } \\
\text { blood stasis; Diuretics; } \\
\text { Anti-dysmenorrhea }\end{array}$ \\
\hline 61 & Yebaihe & $\begin{array}{l}\text { 野 } \\
\text { 百 } \\
\text { 合 }\end{array}$ & $n u^{33} \mathrm{pa}^{43}$ & $\begin{array}{l}\text { Lilium brownii F.E. } \\
\text { Brown ex Miell. }\end{array}$ & Liliaceae & Herb & Bulb & $* * * *$ & $\begin{array}{l}\text { Moistening lung to } \\
\text { arrest cough; Heat- } \\
\text { clearing and detoxifying } \\
\text { effect; Tranquilizing the } \\
\text { mind; Diuretics; The } \\
\text { bulb is edible }\end{array}$ \\
\hline 62 & Shanjijiao & $\begin{array}{l}\text { 山 } \\
\text { 鸡 } \\
\text { 椒 }\end{array}$ & $\operatorname{siu}^{43}$ & $\begin{array}{l}\text { Litsea cubeba } \\
\text { (Lour.) Pers. }\end{array}$ & Lauraceae & Shrub & Leaf & $* * * * *$ & $\begin{array}{l}\text { Warming spleen and } \\
\text { stomach for dispelling } \\
\text { cold; Dispelling wind } \\
\text { and cold; Anti-swelling } \\
\text { agent; Spice }\end{array}$ \\
\hline 63 & Liuyerendong & $\begin{array}{l}\text { 柳 } \\
\text { 叶 } \\
\text { 忍 } \\
\text { 冬 }\end{array}$ & $\mathrm{ha}^{33} \mathrm{tiau}^{33} \mathrm{tci}^{33} \operatorname{tin}^{43} \mathrm{k}^{33}$ & $\begin{array}{l}\text { Lonicera } \\
\text { lanceolata Wall. }\end{array}$ & Caprifoliaceae & Tree & $\begin{array}{l}\text { Stem, } \\
\text { Leaf, } \\
\text { flower }\end{array}$ & $* * * *$ & None \\
\hline 64 & Danzhuye & $\begin{array}{l}\text { 淡 } \\
\text { 竹 } \\
\text { 叶 }\end{array}$ & $w a^{33} \operatorname{fan}^{55}$ & $\begin{array}{l}\text { Lophatherum } \\
\text { gracile Brongn. }\end{array}$ & Poaceae & Herb & $\begin{array}{l}\text { Whole } \\
\text { plant }\end{array}$ & $* * * * *$ & $\begin{array}{l}\text { Reducing pathogenic } \\
\text { fire; Fever; Diuretics; } \\
\text { Herbal tea }\end{array}$ \\
\hline
\end{tabular}


Table 1 Plants used for liquor fermentation starter in Sandu County of Guizhou (Continued)

\begin{tabular}{|c|c|c|c|c|c|c|c|c|c|}
\hline 65 & Haijinsha & $\begin{array}{l}\text { 海 } \\
\text { 金 } \\
\text { 沙 }\end{array}$ & $\operatorname{miau}^{33} \mathrm{nu}^{33} \mathrm{ka}^{55}$ & $\begin{array}{l}\text { Lygodium } \\
\text { japonicum } \\
\text { (Thunb.) Sw. }\end{array}$ & Lygodiaceae & Herb & $\begin{array}{l}\text { Aerial } \\
\text { part }\end{array}$ & $* * * * *$ & $\begin{array}{l}\text { Clearing away heat and } \\
\text { toxic materials; } \\
\text { Removing dampness; } \\
\text { Relieving pain Urinary } \\
\text { tract infections; } \\
\text { Hepatitis; Nephritis } \\
\text { edema; Diarrhea }\end{array}$ \\
\hline 66 & $\begin{array}{l}\text { Xiaoguo- } \\
\text { shidagonglao }\end{array}$ & $\begin{array}{l}\text { 小 } \\
\text { 果 } \\
\text { 十 } \\
\text { 大 } \\
\text { 功 } \\
\text { 劳 }\end{array}$ & $\operatorname{lan}^{33} \operatorname{ta}^{33} \operatorname{sun}^{33} \operatorname{ta}^{33} \mathrm{kun}^{33} \operatorname{lau}^{24}$ & $\begin{array}{l}\text { Mahonia } \\
\text { bodinieri Gagnep. }\end{array}$ & Berberidaceae & Shrub & $\begin{array}{l}\text { Whole } \\
\text { plant }\end{array}$ & $* * * * *$ & $\begin{array}{l}\text { Clearing away heat and } \\
\text { toxic materials; Anti- } \\
\text { swelling agent; Antidiar- } \\
\text { rheic; Dysentery; Hepa- } \\
\text { titis; Ornamental }\end{array}$ \\
\hline 67 & Diren & $\begin{array}{l}\text { 地 } \\
\text { 菍 }\end{array}$ & $\mid a i^{33} z^{24}$ & $\begin{array}{l}\text { Melastoma } \\
\text { dodecandrum } \\
\text { Lour. }\end{array}$ & Melastomaceae & Shrub & $\begin{array}{l}\text { Aerial } \\
\text { part }\end{array}$ & $* * * * *$ & $\begin{array}{l}\text { Removing toxicity; The } \\
\text { fruit is edible }\end{array}$ \\
\hline 68 & Shiqizhu & $\begin{array}{l}\text { 石 } \\
\text { 荠 } \\
\text { 茊 }\end{array}$ & $\operatorname{tin}^{43} \operatorname{tci}^{33} \operatorname{han}^{24}$ & $\begin{array}{l}\text { Mosla scabra } \\
\text { (Thunb.) C.Y. Wu } \\
\text { \& H.W. Li }\end{array}$ & Lamiaceae & Herb & $\begin{array}{l}\text { Aerial } \\
\text { part }\end{array}$ & $* * * * *$ & $\begin{array}{l}\text { Cold; Malaria; } \\
\text { Constipation; Hemafecia; } \\
\text { Bleeding; Traumatic } \\
\text { injury }\end{array}$ \\
\hline 69 & Yangmei & $\begin{array}{l}\text { 杨 } \\
\text { 梅 }\end{array}$ & hai $^{24}$ & $\begin{array}{l}\text { Myrica rubra } \\
\text { (Lour.) Sieb. \& } \\
\text { Zucc. }\end{array}$ & Myricaceae & Tree & $\begin{array}{l}\text { Leaf, } \\
\text { Fruit }\end{array}$ & $* * * * *$ & $\begin{array}{l}\text { Inducing saliva and } \\
\text { slakes thirst; Digestion; } \\
\text { The fruit is edible }\end{array}$ \\
\hline 70 & Shenjue & $\begin{array}{l}\text { 肾 } \\
\text { 蔴 }\end{array}$ & $\mathbf{n}_{\mathbf{0}} \mathrm{i}^{33} \operatorname{tchian}^{43}$ & $\begin{array}{l}\text { Nephrolepis } \\
\text { biserrata (Sw.) } \\
\text { Schott }\end{array}$ & Davalliaceae & Herb & $\begin{array}{l}\text { Whole } \\
\text { plant }\end{array}$ & $* * * * *$ & $\begin{array}{l}\text { Cold; Fever; Cough; } \\
\text { Tuberculosis; Dysentery; } \\
\text { Enteritis; The tuber is } \\
\text { edible }\end{array}$ \\
\hline 71 & Xiaohuaihua & $\begin{array}{l}\text { 小 } \\
\text { 槐 } \\
\text { 花 }\end{array}$ & $\mathrm{nu}^{33} \mathrm{mei}^{33} \operatorname{sun}^{43}$ & $\begin{array}{l}\text { Ohwia caudata } \\
\text { (Thunb.) H. } \\
\text { Ohashi }\end{array}$ & Fabaceae & Shrub & Leaf & $* * * * *$ & Hepatoprotective \\
\hline 72 & $\begin{array}{l}\text { Yezhiwei- } \\
\text { jinfenjue }\end{array}$ & $\begin{array}{l}\text { 野 } \\
\text { 雉 } \\
\text { 尾 } \\
\text { 金 } \\
\text { 粉 } \\
\text { 蔴 }\end{array}$ & $\mathrm{n}_{\mathbf{o}} \mathrm{in}^{24} \mathrm{ja}^{33} \mathrm{to}^{24}$ & $\begin{array}{l}\text { Onychium } \\
\text { japonicum } \\
\text { (Thunb.) Kze. }\end{array}$ & Pteridaceae & Herb & $\begin{array}{l}\text { Aerial } \\
\text { part }\end{array}$ & $* * *$ & $\begin{array}{l}\text { Clearing away heat and } \\
\text { toxic materials; } \\
\text { Removing dampness; } \\
\text { Stopping bleed; The } \\
\text { leaves are cooked as } \\
\text { vegetables }\end{array}$ \\
\hline 73 & Jishiteng & $\begin{array}{l}\text { 鸡 } \\
\text { 矢 } \\
\text { 藤 }\end{array}$ & $\mathrm{jau}^{33} \mathrm{kai}^{24}$ & $\begin{array}{l}\text { Paederia foetida } \\
\text { L. }\end{array}$ & Rubiaceae & Liana & $\begin{array}{l}\text { Aerial } \\
\text { part }\end{array}$ & $* * * * *$ & $\begin{array}{l}\text { Dispelling wind and } \\
\text { cold; Removing } \\
\text { dampness; Digestion; } \\
\text { Clearing away heat and } \\
\text { toxic materials; Anti- } \\
\text { swelling agent; Promot- } \\
\text { ing blood circulations; } \\
\text { Relieving pain }\end{array}$ \\
\hline 74 & Jinxiangcao & $\begin{array}{l}\text { 锦 } \\
\text { 香 } \\
\text { 草 }\end{array}$ & $\operatorname{lan}^{24} \operatorname{kan}^{43}$ & $\begin{array}{l}\text { Phyllagathis } \\
\text { cavaleriei (Levl. et } \\
\text { Van.) Guillaum. }\end{array}$ & Melastomaceae & Shrub & $\begin{array}{l}\text { Whole } \\
\text { plant }\end{array}$ & $* * * * *$ & $\begin{array}{l}\text { Reducing pathogenic } \\
\text { fire; Tonic }\end{array}$ \\
\hline 75 & $\begin{array}{l}\text { Changmang- } \\
\text { jinxiangcao }\end{array}$ & $\begin{array}{l}\text { 长 } \\
\text { 芒 } \\
\text { 锦 } \\
\text { 香 } \\
\text { 草 }\end{array}$ & $\mathrm{kai}^{24} \operatorname{lan}^{24} \mathrm{kan}^{43}$ & $\begin{array}{l}\text { Phyllagathis } \\
\text { longiradiosa C. } \\
\text { Chen }\end{array}$ & Melastomaceae & Shrub & Leaf & $* * *$ & $\begin{array}{l}\text { Clearing away heat and } \\
\text { toxic materials }\end{array}$ \\
\hline 76 & Shanju & $\begin{array}{l}\text { 山 } \\
\text { 蒟 }\end{array}$ & $\operatorname{man}^{33} \mid u n^{24}$ & $\begin{array}{l}\text { Piper hancei } \\
\text { Maxim. }\end{array}$ & Piperaceae & Liana & $\begin{array}{l}\text { Aerial } \\
\text { part }\end{array}$ & $* * * * *$ & $\begin{array}{l}\text { Dispelling wind and } \\
\text { cold; Removing } \\
\text { dampness; Activating } \\
\text { collaterals }\end{array}$ \\
\hline 77 & Pingcheqian & $\begin{array}{l}\text { 平 } \\
\text { 车 } \\
\text { 前 }\end{array}$ & $\mathrm{ma}^{43} \mathrm{ma}^{35}$ & $\begin{array}{l}\text { Plantago } \\
\text { depressa Willd. }\end{array}$ & Plantaginaceae & Herb & $\begin{array}{l}\text { Aerial } \\
\text { part }\end{array}$ & $* * * * *$ & $\begin{array}{l}\text { Clearing away heat and } \\
\text { toxic materials; Diuretics; } \\
\text { The whole plants is } \\
\text { cooked as vegetables }\end{array}$ \\
\hline 78 & Jiegeng & $\begin{array}{l}\text { 桔 } \\
\text { 梗 }\end{array}$ & $\operatorname{han}^{33} \mathrm{tie}^{24}$ & & Campanulaceae & Herb & Root & $* * * * *$ & $\begin{array}{l}\text { Cough;Eliminating } \\
\text { phlegm; Ornamental }\end{array}$ \\
\hline
\end{tabular}


Table 1 Plants used for liquor fermentation starter in Sandu County of Guizhou (Continued)

\begin{tabular}{|c|c|c|c|c|c|c|c|c|}
\hline & & & $\begin{array}{l}\text { Platycodon } \\
\text { grandiflorus } \\
\text { (Jacq.) A. DC. }\end{array}$ & & & & & \\
\hline 79 & Guazijin & $\begin{array}{l}\text { 瓜 } h a^{33} \gamma^{33} \mathrm{ti}^{33} \\
\text { 子 } \\
\text { 金 }\end{array}$ & $\begin{array}{l}\text { Polygala japonica } \\
\text { Houtt. }\end{array}$ & Polygalaceae & Herb & $\begin{array}{l}\text { Aerial } \\
\text { part }\end{array}$ & $* * * * *$ & $\begin{array}{l}\text { Promoting blood } \\
\text { circulations; Eliminating } \\
\text { stasis to stop pain; } \\
\text { Eliminating phlegm and } \\
\text { relieve cough; Clearing } \\
\text { away heat and toxic } \\
\text { materials; Relieving pain }\end{array}$ \\
\hline 80 & Laliao & $\begin{array}{l}\text { 辣 } \\
\text { 羕 }\end{array}$ & $\begin{array}{l}\text { Polygonum } \\
\text { hydropiper L. }\end{array}$ & Polygonaceae & Herb & $\begin{array}{l}\text { Aerial } \\
\text { part }\end{array}$ & $* * * * *$ & $\begin{array}{l}\text { Clearing away heat and } \\
\text { toxic materials; } \\
\text { Eliminating stasis to } \\
\text { stop pain;stopping } \\
\text { bleed; Dysentery; } \\
\text { Traumatic injury; Spice }\end{array}$ \\
\hline 81 & Machixian & $\begin{array}{l}\text { 马 } \mathrm{ma}^{33} \mathrm{jun}^{33} \mathrm{noie}^{24} \\
\text { 齿 } \\
\text { 苋 }\end{array}$ & $\begin{array}{l}\text { Portulaca } \\
\text { oleracea L. }\end{array}$ & Portulacaceae & Herb & $\begin{array}{l}\text { Aerial } \\
\text { part }\end{array}$ & $* * * * *$ & $\begin{array}{l}\text { Clearing away heat and } \\
\text { toxic materials; } \\
\text { Promoting circulation } \\
\text { and removing stasis; } \\
\text { Anti-swelling agent; The } \\
\text { aerial part is cooked as } \\
\text { vegetabl }\end{array}$ \\
\hline 82 & Shiganzi & $\begin{array}{l}\text { 石 } \operatorname{tin}^{33} \mathrm{kai}^{33} \mathrm{a}^{55} \\
\text { 柑 } \\
\text { 子 }\end{array}$ & $\begin{array}{l}\text { Pothos chinensis } \\
\text { (Raf.) Merr. }\end{array}$ & Araceae & Liana & $\begin{array}{l}\text { Stems, } \\
\text { Leaf }\end{array}$ & $* * * *$ & $\begin{array}{l}\text { Dispelling wind and } \\
\text { cold; Removing } \\
\text { dampness; Promoting } \\
\text { blood circulations; } \\
\text { Eliminating stasis to } \\
\text { stop pain; Digestion; } \\
\text { Cough }\end{array}$ \\
\hline 83 & $\mathrm{Ge}$ & 葛 $p e i^{33}$ hai $^{55}$ & $\begin{array}{l}\text { Pueraria montana } \\
\text { (Lour.) Merr. }\end{array}$ & Fabaceae & Liana & Leaf & $* * * * *$ & $\begin{array}{l}\text { Fever; Inducing saliva } \\
\text { and slakes thirst; } \\
\text { Inducing saliva and } \\
\text { slakes thirst; } \\
\text { Antidiarrheic; Kudzu } \\
\text { powder is edible }\end{array}$ \\
\hline 84 & Wannianqing & $\begin{array}{l}\text { 万 } \operatorname{cin}^{33} \mathrm{pe}^{31} \mathrm{tchiu}^{55} \\
\text { 年 } \\
\text { 青 }\end{array}$ & $\begin{array}{l}\text { Rohdea japonica } \\
\text { (Thunb.) Roth }\end{array}$ & Liliaceae & Herb & Leaf & $* * * *$ & $\begin{array}{l}\text { Clearing away heat and } \\
\text { toxic materials; } \\
\text { Eliminating stasis to } \\
\text { stop pain; Relieving pain }\end{array}$ \\
\hline 85 & Cili & $\begin{array}{l}\text { 刺 } n e i^{24} \\
\text { 梨 }\end{array}$ & $\begin{array}{l}\text { Rosa roxburghii } \\
\text { Tratt. }\end{array}$ & Rosaceae & Shrub & Fruit & $* * * * *$ & $\begin{array}{l}\text { Heat-clearing and } \\
\text { detoxifying effect; } \\
\text { Inducing saliva and } \\
\text { slakes thirst; Digestion; } \\
\text { The fruit is edible }\end{array}$ \\
\hline 86 & Jinyingzi & $\begin{array}{l}\text { 金 } n_{0} y^{33} m_{\partial \eta^{3}} \mathrm{ja}^{43} \\
\text { 樱 } \\
\text { 子 }\end{array}$ & $\begin{array}{l}\text { Rosa laevigata } \\
\text { Michx. }\end{array}$ & Rosaceae & Shrub & $\begin{array}{l}\text { Leaf, } \\
\text { Fruit }\end{array}$ & $* * * * *$ & $\begin{array}{l}\text { Promoting blood } \\
\text { circulations; Eliminating } \\
\text { stasis to stop pain; } \\
\text { Dispelling wind and } \\
\text { cold; Removing } \\
\text { dampness; Clearing } \\
\text { away heat and toxic; } \\
\text { The fruit is } \\
\text { ediblematerials }\end{array}$ \\
\hline 87 & Cuyexuangouzi & $\begin{array}{l}\text { 粗 }\left|u \mathrm{ei}^{31} \mathrm{wa}^{24}\right| \mathrm{au}^{255} \\
\text { 叶 } \\
\text { 悬 } \\
\text { 钩 } \\
\text { 子 }\end{array}$ & $\begin{array}{l}\text { Rubus } \\
\text { alceaefolius Poir. }\end{array}$ & Rosaceae & Shrub & $\begin{array}{l}\text { Root, } \\
\text { Leaf }\end{array}$ & $* * *$ & $\begin{array}{l}\text { Promoting blood } \\
\text { circulations; Removing } \\
\text { blood stasis; Heat- } \\
\text { clearing and detoxifying } \\
\text { effect; Stopping bleed; } \\
\text { The fruit is edible }\end{array}$ \\
\hline 88 & Shanmei & $\begin{array}{l}\text { 山 } \operatorname{tun}^{33} \mathrm{ka}^{255} \\
\text { 莓 }\end{array}$ & $\begin{array}{l}\text { Rubus } \\
\text { corchorifolius L. f. }\end{array}$ & Rosaceae & Shrub & $\begin{array}{l}\text { Stem, } \\
\text { Leaf, } \\
\text { Fruit }\end{array}$ & $* * * *$ & $\begin{array}{l}\text { Promoting blood } \\
\text { circulations; Stopping } \\
\text { bleed; Dispelling wind } \\
\text { and cold; Removing }\end{array}$ \\
\hline
\end{tabular}


Table 1 Plants used for liquor fermentation starter in Sandu County of Guizhou (Continued)

\begin{tabular}{|c|c|c|c|c|c|c|c|c|}
\hline & & & & & & & & $\begin{array}{l}\text { dampness; The fruit is } \\
\text { edible }\end{array}$ \\
\hline 89 & $\begin{array}{l}\text { Tuoyuan- } \\
\text { xuangouzi }\end{array}$ & $\begin{array}{l}\text { 椭 }\left|\mathrm{un}^{33}\right| \mathrm{an}^{24} \mathrm{ku}^{33} \mid \mathrm{u}^{33} \\
\text { 圆 } \\
\text { 悬 } \\
\text { 钩 } \\
\text { 子 }\end{array}$ & $\begin{array}{l}\text { Rubus ellipticus } \\
\text { Sm. }\end{array}$ & Rosaceae & Shrub & $\begin{array}{l}\text { Young } \\
\text { stem, } \\
\text { Leaf }\end{array}$ & $* * *$ & $\begin{array}{l}\text { Anti-swelling agent; } \\
\text { Relieving pain; } \\
\text { Antidiarrheic; The fruit is } \\
\text { edible }\end{array}$ \\
\hline 90 & $\begin{array}{l}\text { Huangguo- } \\
\text { xuangouzi }\end{array}$ & $\begin{array}{l}\text { 黄 } \operatorname{lun}^{33} \operatorname{lan}^{24} \operatorname{man}^{33} \\
\text { 果 } \\
\text { 悬 } \\
\text { 钩 } \\
\text { 子 }\end{array}$ & $\begin{array}{l}\text { Rubus } \\
\text { xanthocarpus } \\
\text { Bureau \& Franch. }\end{array}$ & Rosaceae & Shrub & Leaf & $* * *$ & $\begin{array}{l}\text { Anti-inflammatory } \\
\text { effects; Relieving pain; } \\
\text { The fruit is edible }\end{array}$ \\
\hline 91 & Hongpaociteng & $\begin{array}{l}\text { 红 } \mathrm{jau}^{43} \mid \mathrm{un}^{33} \mathrm{han}^{33} \\
\text { 泡 } \\
\text { 刺 } \\
\text { 藤 }\end{array}$ & $\begin{array}{l}\text { Rubus niveus } \\
\text { Thunb. }\end{array}$ & Rosaceae & Shrub & $\begin{array}{l}\text { Leaf, } \\
\text { Fruit }\end{array}$ & $* * * *$ & $\begin{array}{l}\text { Dispelling wind and } \\
\text { cold; Removing } \\
\text { dampness; Clearing } \\
\text { away heat and toxic } \\
\text { materials; Dysentery; } \\
\text { The fruit is edible }\end{array}$ \\
\hline 92 & Daxueteng & $\begin{array}{l}\text { 大 } m a^{31} \operatorname{len}^{24} t_{6 i} e^{255} \\
\text { 血. } \\
\text { 藤 }\end{array}$ & $\begin{array}{l}\text { Sargentodoxa } \\
\text { cuneata (Oliv.) } \\
\text { Rehder \& E.H. } \\
\text { Wilson }\end{array}$ & Lardizabalaceae & Liana & $\begin{array}{l}\text { Stem, } \\
\text { Leaf }\end{array}$ & $* * * *$ & $\begin{array}{l}\text { Clearing away heat and } \\
\text { toxic materials; } \\
\text { Promoting blood } \\
\text { circulations; Dredging } \\
\text { collaterals; Dispelling } \\
\text { wind and cold; } \\
\text { Convulsive disease }\end{array}$ \\
\hline 93 & Xiaoxueteng & $\begin{array}{l}\text { 小 } \mathrm{ma}^{31} \mathrm{jiu}^{43} \mathrm{t}_{\mathrm{cie}} \mathrm{e}^{55} \\
\text { 血. } \\
\text { 藤 }\end{array}$ & $\begin{array}{l}\text { Schisandra } \\
\text { propinqua (Wall.) } \\
\text { Baillon }\end{array}$ & Schisandraceae & Liana & $\begin{array}{l}\text { Aerial } \\
\text { part }\end{array}$ & $* * * *$ & $\begin{array}{l}\text { Clearing away heat and } \\
\text { toxic materials; Anti- } \\
\text { swelling agent; Eliminat- } \\
\text { ing stasis to stop pain; } \\
\text { Stopping bleed }\end{array}$ \\
\hline 94 & Tiegusan & $\begin{array}{l}\text { 铁 } \sin ^{33} \mathrm{nuei}^{33} \mathrm{ku}^{33} \operatorname{san}^{33} \\
\text { 䈐 } \\
\text { 散 }\end{array}$ & $\begin{array}{l}\text { Schisandra } \\
\text { propinqua subsp. } \\
\text { sinensis (Oliv.) } \\
\text { R.M.K. Saunders }\end{array}$ & Schisandraceae & Liana & $\begin{array}{l}\text { Stem, } \\
\text { Leaf, } \\
\text { Fruit }\end{array}$ & $* * * *$ & $\begin{array}{l}\text { Dispelling wind and } \\
\text { cold; Promoting blood } \\
\text { circulations; Clearing } \\
\text { away heat and toxic } \\
\text { materials; Anti-swelling } \\
\text { agent; Relieving pain; Ir- } \\
\text { regular menstruation }\end{array}$ \\
\hline 95 & Sanmaibaqia & 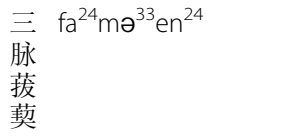 & $\begin{array}{l}\text { Smilax trinervula } \\
\text { Miqual }\end{array}$ & Liliaceae & Shrub & Leaf & $* * * *$ & None \\
\hline 96 & Citianqie & $\begin{array}{l}\text { 刺 } \\
\text { 天 } \\
\text { 天 } \\
\text { 茄 }\end{array}$ & $\begin{array}{l}\text { Solanum } \\
\text { violaceum Ortega }\end{array}$ & Solanaceae & Shrub & Leaf & $* * *$ & $\begin{array}{l}\text { Anti-inflammatory } \\
\text { effects; Clearing away } \\
\text { heat and toxic materials; } \\
\text { Relieving pain }\end{array}$ \\
\hline 97 & Baitan & $\underset{\text { 檀 }}{\text { 白 }} \mathrm{pa}^{33} \mathrm{than}^{24}$ & $\begin{array}{l}\text { Symplocos } \\
\text { paniculata Miq. }\end{array}$ & Symplocaceae & Shrub & Leaf & $* * * * *$ & $\begin{array}{l}\text { Clearing away heat and } \\
\text { toxic materials; } \\
\text { Removing blood stasis; } \\
\text { Dispelling wind and } \\
\text { cold }\end{array}$ \\
\hline 98 & Hongdoushan & $\begin{array}{l}\text { 红 } \mathrm{mei}^{24} \mathrm{nu}^{33} \\
\text { 豆 } \\
\text { 杉 }\end{array}$ & $\begin{array}{l}\text { Taxus wallichiana } \\
\text { Zucc. var. } \\
\text { chinensis (Pilg.) } \\
\text { Florin }\end{array}$ & Taxaceae & Tree & $\begin{array}{l}\text { Young } \\
\text { leaf }\end{array}$ & $* * * * *$ & $\begin{array}{l}\text { Digestion; Ascariasis; } \\
\text { Ornamental }\end{array}$ \\
\hline 99 & Qinglichai & $\begin{array}{l}\text { 青 } \\
\text { 篱 } \\
\text { 柴 }\end{array}$ & $\begin{array}{l}\text { Tirpitzia sinensis } \\
\text { (Hemsl.) Hallier f. }\end{array}$ & Linaceae & Shrub & $\begin{array}{l}\text { Stem, } \\
\text { leaf }\end{array}$ & $* * *$ & $\begin{array}{l}\text { Anti-swelling agent; } \\
\text { Relieving pain; Bone } \\
\text { fracture; Ornamental }\end{array}$ \\
\hline 100 & Xiangchun & $\begin{array}{l}\text { 香 } \mathrm{mei}^{24} \mathrm{n}_{\mathbf{o}} \mathrm{iu}^{43} \\
\text { 椿 }\end{array}$ & $\begin{array}{l}\text { Toona sinensis (A. } \\
\text { Juss.) M. Roem. }\end{array}$ & Meliaceae & Tree & Leaf & $* * * * *$ & $\begin{array}{l}\text { Cold; Removing } \\
\text { dampness; } \\
\text { Stomachache; } \\
\text { Dysentery; The leaves is } \\
\text { cooked as vegetable }\end{array}$ \\
\hline
\end{tabular}


Table 1 Plants used for liquor fermentation starter in Sandu County of Guizhou (Continued)

\begin{tabular}{|c|c|c|c|c|c|c|c|c|}
\hline 101 & Mabiancao & $\begin{array}{l}\text { 马 } \mathrm{ma}^{24} \mathrm{pian}^{33} \mathrm{kan}^{43} \\
\text { 鞭 } \\
\text { 草 }\end{array}$ & $\begin{array}{l}\text { Verbena officinalis } \\
\text { L. }\end{array}$ & Verbenaceae & Herb & $\begin{array}{l}\text { Aerial } \\
\text { part }\end{array}$ & $* * * * *$ & $\begin{array}{l}\text { Promoting blood } \\
\text { circulations; Eliminating } \\
\text { stasis to stop pain; } \\
\text { Malaria; Clearing away } \\
\text { heat and toxic materials; } \\
\text { Diuretics; Anti-swelling } \\
\text { agent }\end{array}$ \\
\hline 102 & Lanshu & $\begin{array}{l}\text { 蓝 } \text { mei }^{24} \text { tchiu } \\
\text { 树 }\end{array}$ & $\begin{array}{l}\text { Wrightia laevis } \\
\text { Hook. f. }\end{array}$ & Apocynaceae & Tree & Leaf & $* * * * *$ & $\begin{array}{l}\text { Traumatic injury; } \\
\text { Stopping bleed; Dye } \\
\text { plant }\end{array}$ \\
\hline 103 & Huajiao & $\begin{array}{l}\text { 花 } \operatorname{ciu}^{24} \\
\text { 椒 }\end{array}$ & $\begin{array}{l}\text { Zanthoxylum } \\
\text { bungeanum } \\
\text { Maxim. }\end{array}$ & Rutaceae & Tree & Leaf & $* * * * *$ & $\begin{array}{l}\text { Digestion; Relieving } \\
\text { pain; Insecticidal; Anti- } \\
\text { itch; Spice }\end{array}$ \\
\hline
\end{tabular}

Frequency: ${ }^{* * * *}>75 \%$ of respondents; ${ }^{* * * *}>50 \%$ of respondents; ${ }^{* * *}>25 \%$ of respondents; ${ }^{* *}>12.5 \%$ of respondents; ${ }^{*}<12.5 \%$ of respondents, but at least 5 respondents

(Ranked by scientific names alphabetically, followed by generic and species names)

reported plants is to clear heat away followed by detoxification. Some plants had a single medicinal function while others had multiple medicinal functions. For example, Lygodium japonicum is used to treat urinary tract infections, hepatitis, nephritis edema, diarrhea, and other health conditions while Melastoma dodecandrum is used for removing toxicity. A few documented species are used for both food and medicine such as Litsea cubeba, Imperata cylindrica, Kadsura longipedunculata, Cinnamomum cassia, Codonopsis pilosula, Ficus pumila and Rosa roxburghii. In addition to food and medicinal purposes and for making starters, the documented species are valued for providing household income, celebrating local festivals and for construction material. For example, Paederia foetida is used for making festival rice cakes by the Shui and Cunninghamia lanceolata is the main timber tree species used for local construction.

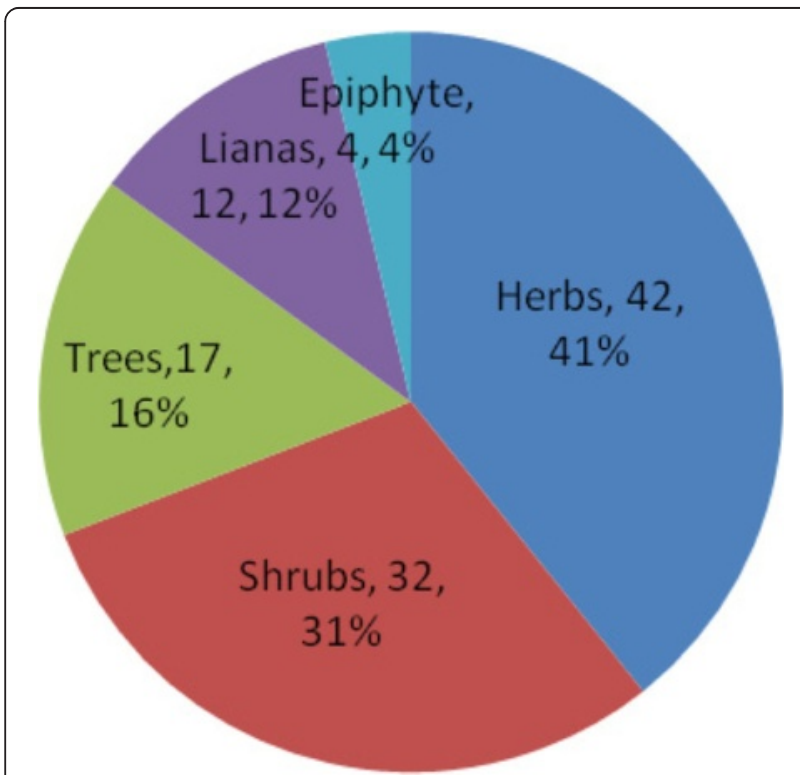

Fig. 4 Life forms of wild plants used for liquor fermentation starter
Wrightia laevis is used to dye the customary clothing of the Shui and results in a dark blue stain that is valued for clothing by Shui informants (Fig. 2).

Informants reported that fermented alcoholic beverages prepared from wild plants had enhanced taste that is smoother compared to beverages prepared without these plants. Most informants reported that using a greater number of plant species as a starter results in improved quality of the final fermented alcoholic beverage. In addition, informants reported that wild species are conveniently located near households in the study area and are available free of cost. Nowadays, informants travel several dozens of kilometers to procure these plants as not all plants used are located near their households and because of habitat destruction near their households. Compared to cultivated plants, wild plants require less management, are usually not directly impacted by agro-chemical pollution, and are a rich source of micronutrients [27]. Interviews and participant observation in local fields $[31,32]$ found that none of the documented plants were cultivated. In addition, participant observation in local markets [33] found that none of the documented plants were sold for liquor fermentation.

\section{Traditional harvest and preparation of Jiuqianjiu}

Shui families are able to provide the daily quantity and quality of Jiuqianjiu liquor needed from locally available resources that are relatively cheap as well as with the use of simple equipment. Given the accessibility of needed plant material and affordability of equipment in making fermented alcoholic beverages, Shui women can support the social life of their families with this traditional practice. Preparation starts with harvesting wild plants. Harvested plants are usually dried in the sun after collection and are then powdered and stored in a cool dry place for preservation of flavor and health attributes. 


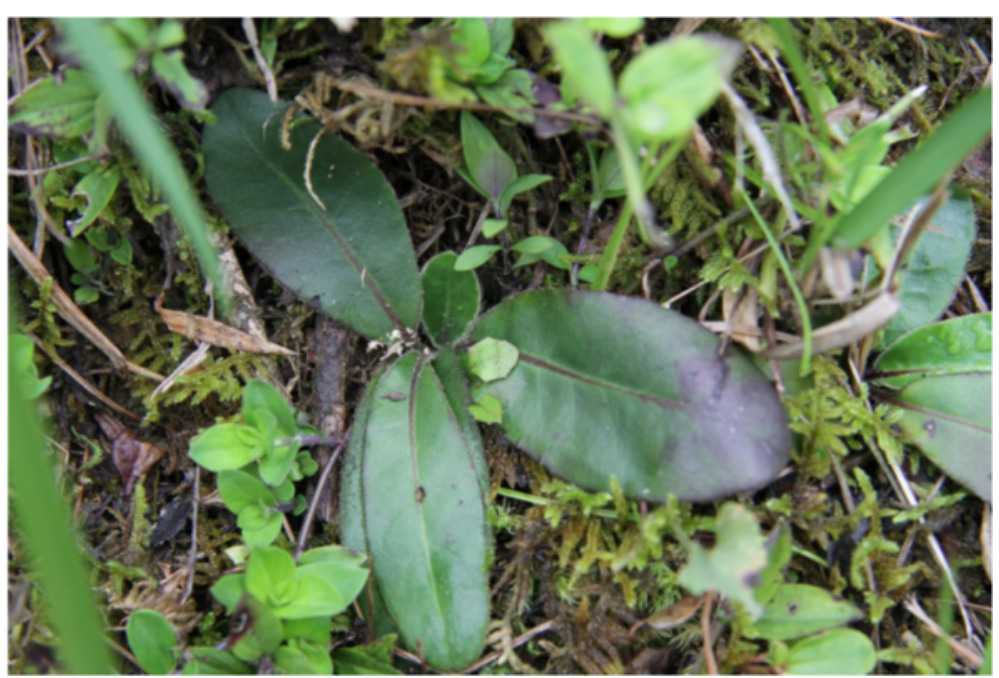

Fig. 5 Gerbera piloselloides

The Shui have developed their traditional production sequence of liquor-making according to their farming seasons. Wild plants for starters are harvested between May to September in the study areas. Usually, local households make the beverage starter in summer and brew the liquor during the autumn. The Shui practice of preparing starters in the summer in congruent to previous studies that widely supported that microorganisms bred more quickly in summer and thereby shorten the production cycle and provided greater liquor yield [34, 35]. That is because the temperature in summer is believed to be better due to certain criteria, such as rapid fermentation.

Locals harvest wild plants according to local sociallynegotiated protocols that prevent overharvesting of common resources including only harvesting mature individuals when whole plants are needed. In addition, locals prepare enough liquor fermentation starter at one time for three years to ensure plenty of time for plant growth. Local practices of regulating harvesting of common wild plant resources and making enough liquor starter at a time for three years to allow time for plant growth highlights the sustainability ethos of local communities. It is vital to conserve these wild plants while protecting their habitats. They must also be managed in sustainable ways to promote their use in suitable method [36]. It is suggested recruiting ethnobotanists and experts to train local communities on the sustainable utilization of wild plant resources to complement traditional practices [37, 38].

Both non-sticky rice and sticky rice are used by informants as the primary ingredient of Jiuqianjiu liquor with

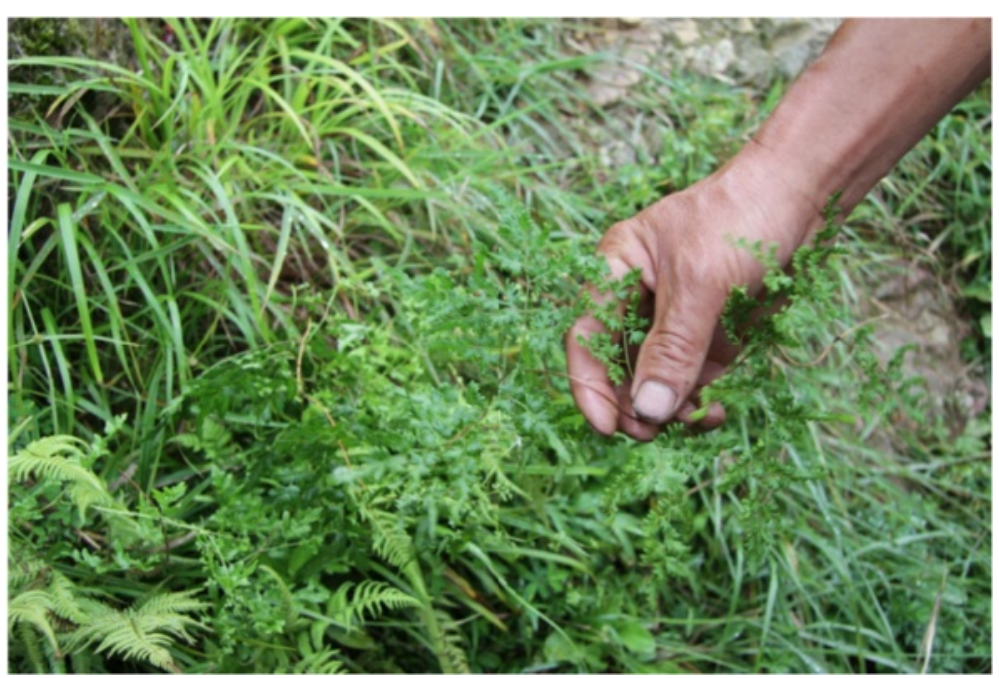

Fig. 6 Lygodium japonicum 


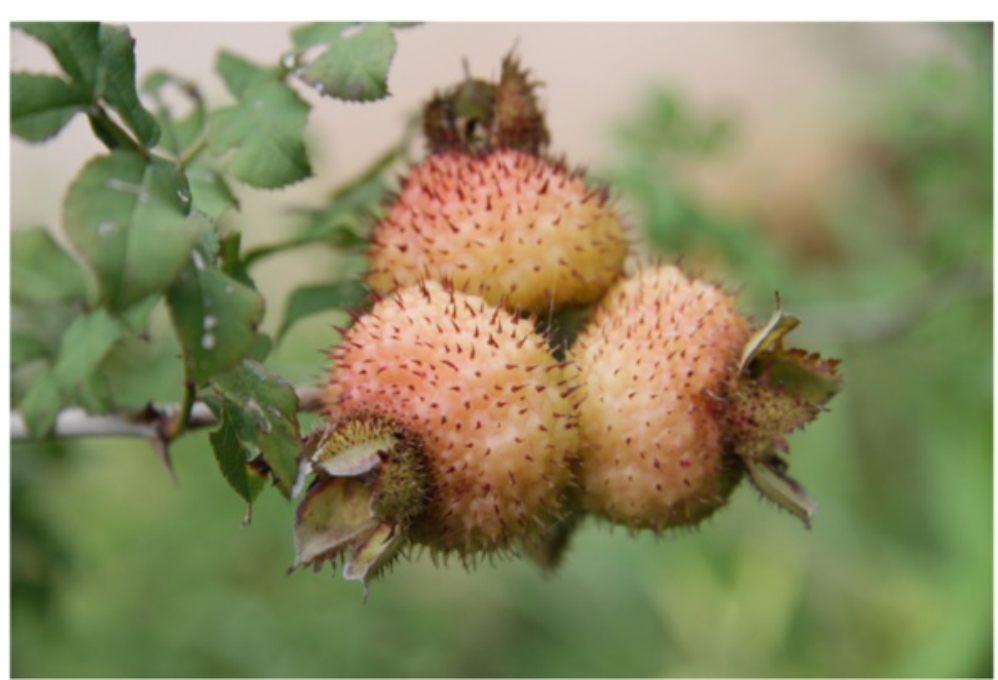

Fig. 7 Rosa roxburgii

Xiaoqu being the starter agent during the fermentation process. Glutinous rice is the favored rice for liquor brewing by Shui informants because locals perceive it results in a higher quality product that has better taste. There are 15 glutinous rice varieties in Shui communities in Sandu County compared to 7 varieties in other areas of Sandu County. However, the lower yield of glutinous rice results in a relatively higher cost than common rice in the process of liquor making. These glutinous rice varieties are generally reserved for use in festival foods and desserts as well as serving as the main raw material for liquor making in the study area. Locals especially value black glutinous rice. Previous nutrition analysis and phytochemical investigation on black glutinous rice has relatively higher levels of polyphenols and anthocyanin content compared to other varieties of rice; these compounds are known for their medicinal functions in humans including antioxidant activity, reducing cholesterol levels and inhibiting cancer cell proliferation [39-44]. In addition, black glutinous rice has a number of nutritional advantages compared to many other rice varieties including higher content of protein, vitamins and minerals [45].

Polished rice is used for preparing Jiuqianjiu liquor that is thought to remove substances in rice aside from the starch that are regarded as undesirable substances for liquor brewing. Following polishing, the rice is washed and immerged using cold spring water at room temperature for $24 \mathrm{~h}$. After steeping, excess water is drained off for $4 \mathrm{~h}$ before cooking the rice with steam for $1 \mathrm{~h}$ in a wood rice steamer. Subsequently, the

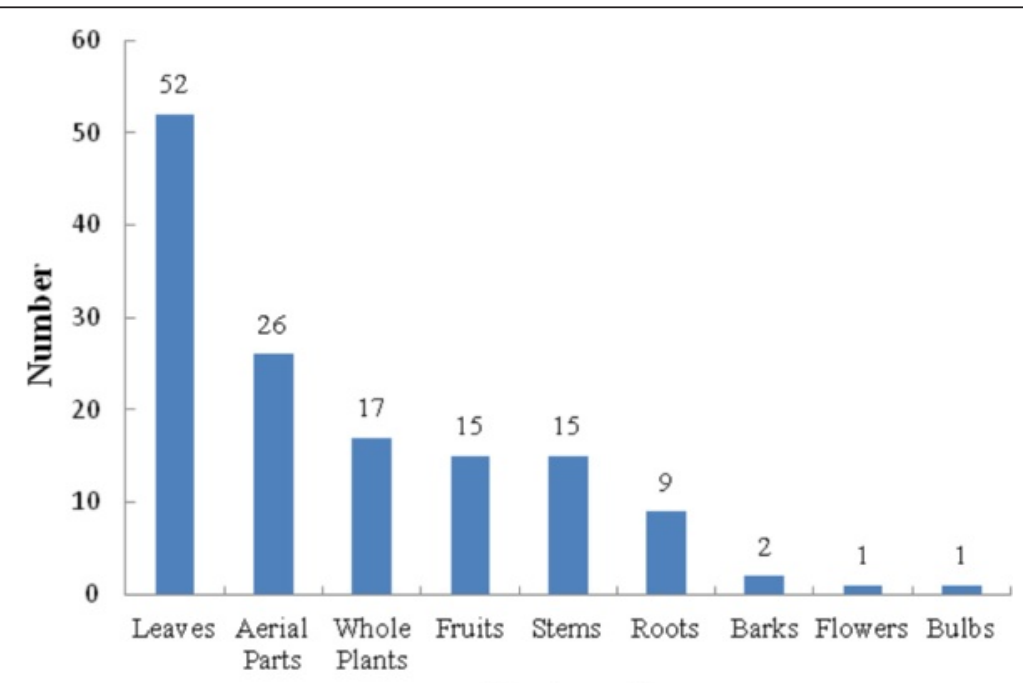

\section{Parts used}

Fig. 8 Plant parts used for liquor fermented starter 
Table 2 Types of multiple uses for wild plants in Sandu County of Guizhou

\begin{tabular}{lcc}
\hline Value & Number & Percentage (\%) \\
\hline beverage starter & 103 & 100.0 \\
Medicinal & 96 & 93.2 \\
Edible & 30 & 29.1 \\
Ornamental & 11 & 10.7 \\
Spice & 4 & 3.9 \\
Construction & 2 & 1.9 \\
Herbal tea & 2 & 1.9 \\
Fence & 1 & 1.0 \\
Dyeing & 1 & 1.0 \\
\hline
\end{tabular}

steamed rice is spread out for cooling in the open air until the temperature falls to nearly $35^{\circ} \mathrm{C}$.

Beverage starters usually occur as a dried ball or cake of flour cultured with various molds, yeasts and bacteria (Fig. 9). Xiaoqu, which is the dominant starter for Jiuqianjiu liquor at the study sites, is prepared by a wild type microbe inoculation of molds, yeasts and bacteria as well as their growth on rice or other grains. The starter is crushed and added to inoculate the cereal substrate to initiate fermentation into liquor.

Informants reported a general method for mixing material for starters that includes boiling the plant mixture powder $(5 \%, \mathrm{w} / \mathrm{w})$ with the spring water $(48 \%, \mathrm{w} / \mathrm{w})$ for $3 \mathrm{~h}$ and then mixing the rice bran and glutinous rice flour $(45 \%, w / w)$ with the last year's starter $(2 \%, w / w)$. The grain mix is then pulverized and the plant materials are stirred in. A wild type microbe inoculation method is used to promote the growth of molds, yeasts and other bacteria. The mixed material is typically milled and pressed into a mould of egg-size by hand. Then, it is incubated at 28-30 ${ }^{\circ} \mathrm{C}$ for 7 days in a room with special climatic conditions. After 7 days of incubation the mixture is dried at $45{ }^{\circ} \mathrm{C}$ until the humidity is lower than $15 \%(\mathrm{w} / \mathrm{w})$. The starter is then stored in a cold and dry place until use for liquor making within the next three years.

The steamed rice is mixed with $1 \%(\mathrm{w} / \mathrm{w})$ of the starter mixture that starts the processes of saccharification, acidification, and fermentation of the steamed rice. Then, the mixture is placed in wooden cask that is placed in a pit dug in the ground at $40{ }^{\circ} \mathrm{C}$ for 2 days. The saccharified mixture is then mixed with $120 \%(\mathrm{w} / \mathrm{w})$ cold spring water to form the thick slurry. Subsequently, the slurry is fermented at room temperature for 15 days in a semi-solid state. This slurry is carefully stirred by local liquor makers to aerate and maintain an optimal level of oxygen and carbon dioxide in the mixture, as well as to maintain an even temperature throughout the fermenting process. After fermentation, the rice wine mash is filtered using a bamboo basket for the purpose of removing the fermented grains. Then, a part of filtered liquid is evaporated to the dense liquid while the other is used for distilling the liquor. The two parts are then mixed at a certain proportion to increase the concentration of alcohol in the mash and halt the fermentation process.

The process described above (Fig. 10) leaves a notable quantity of unfermented saccharides and chemical compounds from the plants and rice, thus producing a sweet taste and mouth-feel that is distinct from other forms of rice wine. Finally, the slightly turbid rice liquor is pumped through pottery casks for clarification and storage. Usually, the liquor is aged and stored underground for several years at a storage temperature of $13-18{ }^{\circ} \mathrm{C}$. Unlike common rice wine, Jiuqianjiu liquor is distilled and mixed with the fermentation concentrate resulting from the

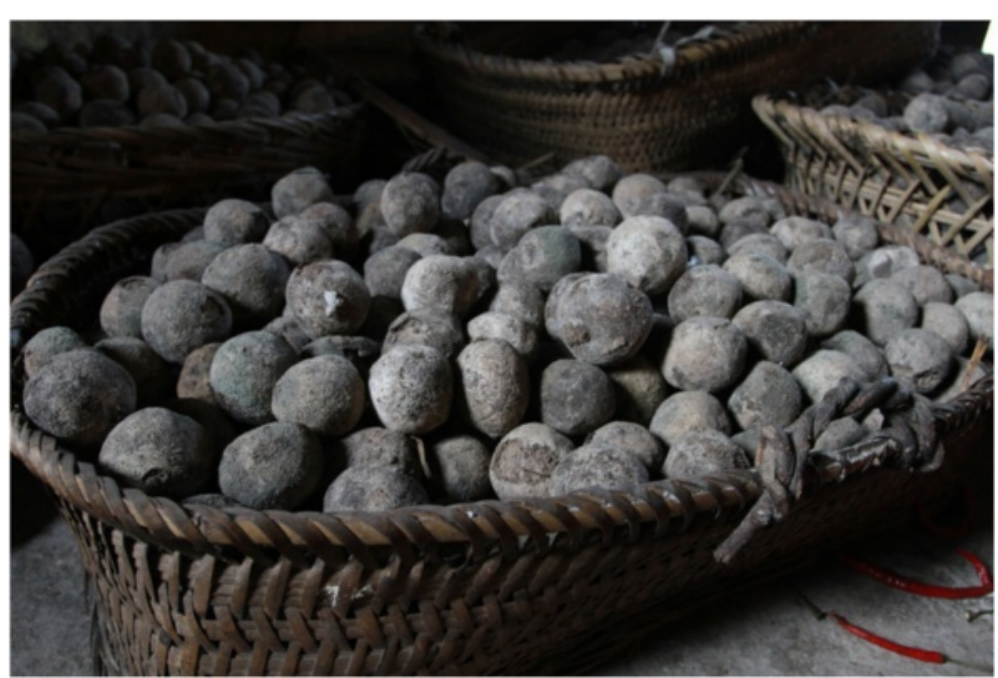

Fig. 9 The liquor fermentation starter, or Xiqoqu starter 


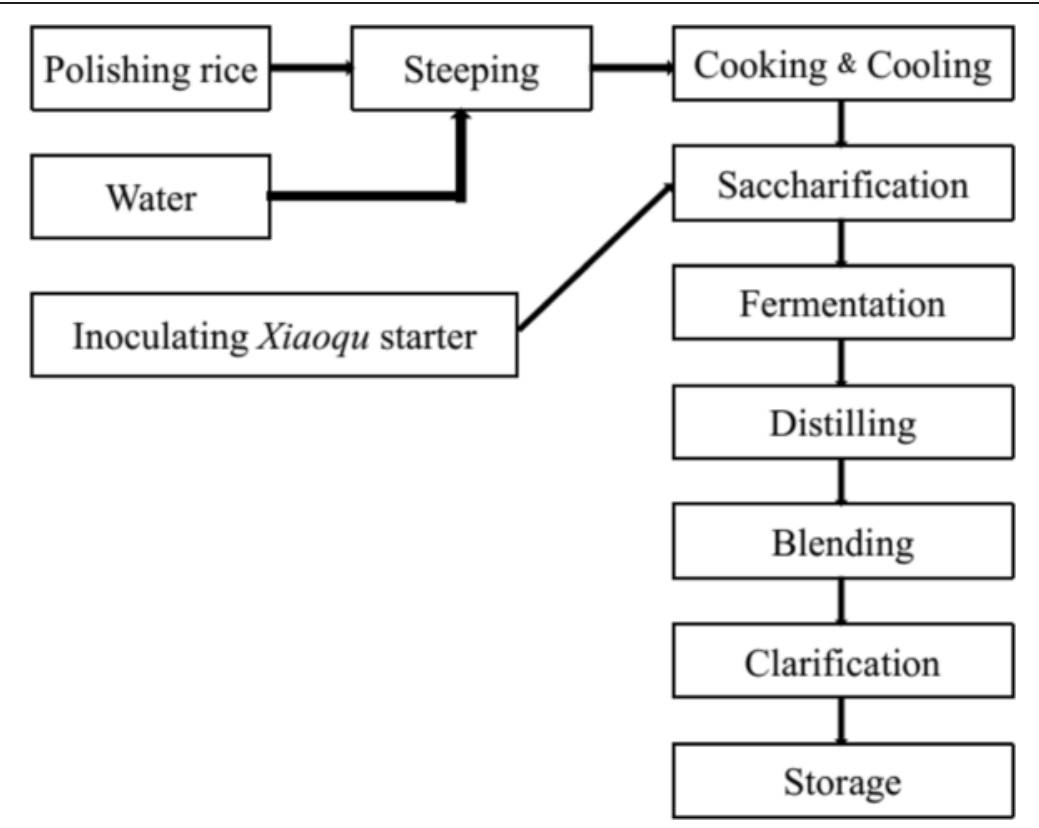

Fig. 10 The processing procedures of Jiuqianjiu liquor in Sandu County of Guizhou

starter. During storage, the liquor matures gradually and adopts a smoother taste. The various types of Jiuqianjiu liquor at the study sites vary in color from beige to yellowish-brown depending on the weight ratio of the distilled liquor versus the fermented concentrate. The final product is consumed at room temperature or after being warmed. Grains spent during fermentation are usually used as fodder for livestock at the study sites.

\section{Shui drinking culture and use of Jiuqianjiu}

Preparation and consumption of Jiuqianjiu liquor is an important part of traditional social life and interpersonal relations at the study sites. There is a cultural practice to socially consume fermented alcoholic beverages in Shui communities that is known as zhuan zhuan jiu. This practice proceeds by all participants having a pot filled with local liquor on the right hand and feeding this beverage to their neighbor to the right. Informants reported that this practice reflects their respect to guests and is offered as a gesture of equality between the hosts and the guests.

Another use involving Jiuqianjiu liquor is by mothers after giving birth to improve their postpartum recovery. After the third day, mothers can go back to work outdoors if they drank Jiuqianjiu liquor after delivery. In comparison, mothers are commonly not allowed to work until 1 month after birth in China. All the informants indicated that traditional Jiuqianjiu liquor can make women during their puerperium (approximately 6 weeks after birth) and their babies healthy and strong. Informants believe that wild plants play a role in this function. This finding of the role of wild plants after birth is consistent with the findings of Yao people in China, whereas the difference is the mode of administration between Yao people and Shui people. Yao people use medicinal plants for medicine bath whereas the Shui consume these plants via fermented alcoholic beverages $[38,46,47]$.

\section{Gender and knowledge transmission}

Women are the primary harvesters of plants used as starters for making Jiuqianjiu liquor as well as the makers of these beverages at the study sites. While most of the male Shui informants carry out housework such as cooking, few male informants had knowledge of how to collect plants for starters and prepare fermented alcoholic beverages. Knowledge of plants used as starters is orally transferred from mother to daughter.

Findings show a significant correlation between informant age and plant knowledge. The distribution of informants in age, gender and education class is shown in Table 3. This study found that most informants who were known to have substantive knowledge on making of Jiuqianjiu liquor and fermented beverages as well as those that were practicing this tradition were primarily older than 50 years old. Female informants represented $78.5 \%$ of the total sample group and the majority of key informants interviewed were females above 65 years old that had little to no formal education. Informants indicated that an increasing amount of young girls studied in school and did not learn the traditional practice of liquor making. This study further showed that 
Table 3 Demographic profile of informants

\begin{tabular}{lll}
\hline Indicator & Description & Frequency (\%) \\
\hline Age & $20-29$ & $7(4.7)$ \\
& $30-39$ & $23(15.4)$ \\
$40-49$ & $28(18.8)$ \\
$50-59$ & $41(27.5)$ \\
$60-69$ & $27(18.1)$ \\
$70-79$ & $15(10.1)$ \\
Gender & $\geq 80$ & $8(5.4)$ \\
Education & Male & $32(21.5)$ \\
& Female & $117(78.5)$ \\
& None & $31(20.8)$ \\
& Primary & $85(57.0)$ \\
& Secondary & $21(14.1)$ \\
& Tertiary & $12(8.1)$
\end{tabular}

female Shui informants who were well educated and aged between 20 and 35 knew how to brew Jiuqianjiu liquor but were not able to identify wild plants used as a beverage starter. Most young females at the study sites no longer learn about these plants and how to prepare traditional fermented beverages; rather, they purchase new commercial starters or fermented alcoholic beverages from the market that are increasingly available. Informants reported that this was mostly because of migration out of rural communities for jobs. Consequently, the traditional Shui ethnobotanical base is failing to be transferred. Knowledge of wild plants should thus be recorded and conveyed in Shui areas for their sustainable use and continuation of cultural practices linked to local biodiversity.

\section{Scale of production}

Shui informants prepare fermented beverages for household consumption and commercially in small-scale local distilleries. Small-scale liquor distilleries are distributed widely throughout the study sites and surrounding areas that provide local people with high-quality and relatively affordable local liquor. Wild plants are collected around residences of producers both for when they are preparing beverages for household use as well as for sale in the market. Jiuqianjiu liquor, like most other local alcoholic beverages in minority areas in China, is still produced with traditional equipment that is not backed by scientific knowledge of the process and has little regard for hygiene. Whereas small-scale manufacture has the advantages of short distribution lines, income generation for families, etc., urbanization and increased regional tourism and the resulting growing demand for ready-toconsume high-quality foods requires larger-scale controlled industrial production.
The final products are dependent on local climatic conditions and therefore the sensory characteristics and the quality are variable. The major problem associated with the traditional method of producing Jiuqianjiu liquor is that the product can never be uniform nor predicted because of the diversity of involved microorganisms and difficulties in controlling this population. Upgrading of traditional home-scale processes is needed so that it will lead to a better standardization and safety of a product that is already acceptable to the cultural tastes of local communities. In addition, this standardization process is helpful to continue to maintain and strengthen cultural heritage of Shui communities while providing a product that can compete successfully with industrial versions. However, Jiuqianjiu liquor is a homemade activity and uncontrolled microbial inoculation based on spontaneous fermentation. The ecosystem in Jiuqianjiu liquor represents a source of biodiversity that can be exploited to create such functional starter cultures. The microbiological details of this highly sophisticated fermentation technology has remained undocumented. In fact, Jiuqianjiu liquor has the potential to become an important source of new valuable microbial strains for biotechnology. Future studies are needed to evaluate the phytochemical profiles, bioactivity, stability and safety of fermented wild plants and their synergies.

Wild plants are threatened by various socio-ecological reasons including climate change, land use change, habitat destruction, overharvesting and etc. [48-52]. The construction of highways and other infrastructure as well as deforestation for agricultural purposes is severely threatening wild habitats for plants in Guizhou Province. In addition, unsustainable harvest of plant species with market value also contributes to a decrease of these wild resources. Both ex situ and in situ conservation methods are needed in the natural or farmed environment to preserve the biodiversity of wild plants used for preparing fermented beverages and associated cultural systems. It is necessary that local people support and participate in these conservation initiatives for the most successful results.

\section{Conclusion}

This paper provides documentation of the diversity of wild plants used as starters to prepare fermented beverages by Shui communities of Sandu County in Southwest China. Findings highlight the rich biodiversity and habitats that local communities draw upon from their surroundings as part of their cultural life to support interpersonal communication and celebrate key occasions. Women's role as the primary producers involved in making fermented beverages reflects gendered knowledge that is related to societal life and relations to kin. While knowledge of plants used for liquor making has traditionally been orally transferred from mother to daughter, this knowledge is threatened as 
the younger generations move away from rural areas in search of jobs and a different lifestyle, a pattern witnessed in rural communities worldwide. Efforts are needed to enhance the transmission of ethnobotancial knowledge in Shui communities towards conservation of biodiversity and associated preservation of cultural systems. Increased interest in natural products and artisanal beverages as well as increased regional tourism is attracting new interest in wild plants used in the processing of fermented foods and beverages. If developed with local community interests and conservation in mind, these commercialization and tourism efforts have the potential of helping preserve traditional ethnobotanical knowledge as well as associated biodiversity. Future studies are needed to evaluate the phytochemical profiles, bioactivity, stability and safety of fermented wild plants and their potential for other fermented foods and beverages as well as medicinal purposes. In addition, it is necessary to develop standards for large-scale production and commercialization of these non-timber forest products. These future studies would help provide guidelines for community-based production and ultimately preservation of biological and cultural diversity.

\section{Consent}

Permissions were provided by all participants in this study, including the Shui people. Consent was obtained from the participants prior to this study being carried out. The authors have all copyrights.

\section{Competing interests}

The authors declare that they have no competing interests.

\section{Authors' contributions}

$\mathrm{LH}$ conducted field work and drafted the manuscript. CL designed the study and contributed to the preparation of the manuscript. SA contributed to discussion regarding the study and to the preparation of the manuscript. CW and $Y L$ made contributions to the discussions. All other authors participated in field surveys and identification of specimens. All authors read and approved the final manuscript.

\section{Acknowledgements \\ We are grateful to Shui people in Sandu County of Guizhou Province for their assistance in the field investigations and for sharing their valuable knowledge. Our research team is also grateful for the numerous discussions and field trips with members of the Ethnobotanical Laboratory at Minzu University of China and the Research Group of Ethnobotany at Kunming Institute of Botany, Chinese Academy of Sciences, and Kaili University, and Guizhou Normal College. We would like to thank Ling Wang from the Department of Minority Languages and Literatures at Minzu University of China for correcting and typing the Shui names of wild plants used for starting fermented beverages. This study was funded by the Ministry of Science \& Technology of China (2012FY110300), the National Natural Science Foundation of China (31161140345 \& 31360070), the Ministry of Education of China and the State Administration of Foreign Experts Affairs (B08044), and Minzu University of China (MUC 10301-01404031-84 \& YLDX10103).}

\section{Author details}

${ }^{1}$ College of Life and Environmental Sciences, Minzu University of China, Beijing 100081, People's Republic of China. ${ }^{2}$ Kunming Institute of Botany, Chinese Academy of Sciences, Kunming 650201, People's Republic of China.

${ }^{3}$ School of Agronomy and Biotechnology, Yunnan Agricultural University,
Kunming 650201, People's Republic of China. ${ }^{4}$ School of Environment \& Life Science, Kaili University, Guizhou 556011, People's Republic of China. 5 Department of Health \& Human Development, Montana State University, Bozeman, MT 59717, USA. '5Chool of Chemistry and Life Science, Guizhou Normal College, Guiyang 550018, People's Republic of China. ${ }^{7}$ Guizhou Institute for Advanced Study in Anthropology \& Ethnology, Guizhou Normal College, Guiyang 550018, People's Republic of China.

Received: 7 January 2015 Accepted: 1 May 2015

Published online: 28 May 2015

\section{References}

1. Schoustra SE, Kasase C, Toarta C, Kassen R, Poulain AJ. Microbial community structure of three traditional Zambian fermented products: mabisi, chibwantu and munkoyo. PLoS One. 2013;8(5):e63948. doi:10.1371/ journal.pone.0063948.

2. Hansen EB. Commercial bacterial starter cultures for fermented foods of the future. Int J Food Microbiol. 2002;78(1):119-31. doi:10.1016/S01681605(02)00238-6

3. Campbell-Platt G. Fermented foods-a world perspective. Food Res Int. 1994;27:253-7.

4. Steinkraus K. Handbook of Indigenous Fermented Foods, revised and expanded. Boca Raton (Florida, USA): CRC Press; 1995

5. Almeida EG, Rachid CCTC, Schwan RF. Microbial population present in fermented beverage 'cauim' produced by Brazilian Amerindians. Int J Food Microbiol. 2007;120(1):146-51. doi:10.1016/j.ijfoodmicro. 2007.06.020

6. Fagbemi AO, ljah UJJ. Microbial population and biochemical changes during production of protein-enriched fufu. World J Microb Biot. 2006;22(6):635-40. doi:10.1007/s11274-005-9083-3.

7. Haruta S, Ueno S, Egawa I, Hashiguchi K, Fujii A, Nagano M, et al. Succession of bacterial and fungal communities during a traditional pot fermentation of rice vinegar assessed by PCR-mediated denaturing gradient gel electrophoresis. Int J Food Microbiol. 2006;109(1):79-87. doi:10.1016/ j.ijfoodmicro.2006.01.015.

8. Schwan RF, Almeida EG, Souza-Dias MAG, Jespersen L. Yeast diversity in rice-cassava fermentations produced by the indigenous Tapirapé people of Brazil. FEMS Yeast Res. 2007;7(6):966-72. doi:10.1111/j.15671364.2007.00241.x

9. Nielsen DS, Teniola OD, Ban-Koffi L, Owusu M, Andersson TS, Holzapfel WH. The microbiology of Ghanaian cocoa fermentations analysed using culturedependent and culture-independent methods. Int J Food Microbiol. 2007;114(2):168-86. doi:10.1016/j.ijfoodmicro.2006.09.010.

10. Swain MR, Anandharaj M, Ray RC, Rani RP. Fermented fruits and vegetables of Asia: a potential source of probiotics. Biotech Res Int 2014 http://dx.doi.org/10.1155/2014/250424

11. Ross RP, Morgan S, Hill C. Preservation and fermentation: past, present and future. Int J Food Microbiol. 2002;79(1):3-16. doi:10.1016/S01681605(02)00174-5.

12. Tamang JP, Sarkar PK, Hesseltine CW. Traditional fermented foods and beverages of Darjeeling and Sikkim-a review. J Sci Food Agri. 1988:44(4):375-85, doi:10.1002/jsfa.2740440410.

13. Blandino A, Al-Aseeri ME, Pandiella SS, Cantero D, Webb C. Cereal-based fermented foods and beverages. Food Res Int. 2003;36(6):527-43. doi:10.1016/S0963-9969(03)00009-7.

14. Sanni Al. The need for process optimization of African fermented foods and beverages. Int J Food Microbiol. 1993;18(2):85-95. doi:10.1016/01681605(93)90213-Z

15. Vieira-Dalodé G, Jespersen L, Hounhouigan J, Moller PL, Nago CM, Jakobsen M. Lactic acid bacteria and yeasts associated with gowé production from sorghum in Bénin. J Appl Microbiol. 2007;103(2):342-9. doi:10.1111/j.13652672.2006.03252.x

16. Montet D, Loiseau G, Zakhia-Rozis N. Microbial technology of fermented vegetables. Micro Biotech Hortic. 2006;1:309-43.

17. Jespersen L. Occurrence and taxonomic characteristics of strains of Saccharomyces cerevisiae predominant in African indigenous fermented foods and beverages. FEMS Yeast Res. 2003:3(2):191-200. doi:10.1016/S1567-1356(02)00185-X

18. Rajalakshmi R, Vanaja K. Chemical and biological evaluation of the effects of fermentation on the nutritive value of foods prepared from rice and grams. Brit J Nutr. 1967;21(02):467-73. doi:http://dx.doi.org/10.1079/BJN19670048. 
19. Shrivastava N, Ananthanarayan L. Use of the backslopping method for accelerated and nutritionally enriched idli fermentation. J Sci Food Agr. 2014. doi:10.1002/jsfa.6923.

20. McGovern PE, Zhang J, Tang J, Zhang ZQ, Hall RG, Moreau AR, et al. Fermented beverages of pre-and proto-historic China. Proc Natl Acad Sci U S A. 2004;101(51):17593-8. doi:10.1073/pnas.0407921102.

21. Edmondson AJ, Esling HJ, Harris GJ, Wei J. A phonetic study of Sui consonants and vowels. Mon-Khmer Studies. 2004:34:47-66.

22. Catford JC. Phonation types: the classification of some laryngeal components of speech production. London: Longman; 1964. p. 26-37.

23. Stanford NJ. "Eating the food of our place": sociolinguistic loyalties in multidialectal Sui villages. Language in Society. 2009;38:287-309. doi:10.1017/S0047404509090502.

24. Alexiades MN, Sheldon JW. Selected Guidelines for Ethnobotanical Research: A Field Manual. New York: Botanical Garden; 1996.

25. Long $\mathrm{CL}$, Wang JR. Participatory rural appraisal: An introduction to principle, methodology and application. Kunming: Yunnan Science and Technology Press; 1996.

26. Yang LX, Ahmed S, Stepp JR, Mi K, Zhao YQ, Ma JZ, et al. Comparative homegarden medical ethnobotany of Naxi healers and farmers in Northwestern Yunnan. China J Ethnobiol Ethnomed. 2014;10(1):6. doi:10.1186/1746-4269-10-6

27. Ju Y, Zhuo JX, Liu B, Long CL. Eating from the wild: diversity of wild edible plants used by Tibetans in Shangri-la region, Yunnan. China J Ethnobiol Ethnomed. 2013:9(1):28. doi:10.1186/1746-4269-9-28.

28. Khasbagan, Huai HY, Pei SJ. Wild plants in the diet of Arhorchin Mongol herdsmen in Inner Mongolia. Econ Bot. 2000;54(4):528-36. doi:10.1007/ BF02866550.

29. Weckerle CS, Huber FK, Yang YP, Sun WB. Plant knowledge of the Shuhi in the Hengduan Mountains, southwest China. Econ Bot. 2006;60(1):3-23. doi:10.1663/0013-0001(2006)60[3:PKOTSI]2.0.CO;2

30. Xu YK, Tao GD, Liu HM, Yan KL, Dao XS. Wild vegetable resources and market survey in Xishuangbanna, southwest China. Econ Bot. 2004;58(4):647-67. doi:10.1663/0013-0001(2004)058[0647:WRAMS]2.0.CO;2

31. Bekalo TH, Woodmatas SD, Woldemariam ZA. An ethnobotanical study of medicinal plants used by local people in the lowlands of Konta Special Woreda, southern nations, nationalities and peoples regional state. Ethiopia J Ethnobiol Ethnomed. 2009:5:26. doi:10.1186/1746-4269-5-26.

32. Nolan JM. The Roots of Tradition: Social Ecology, Cultural Geography, and Medicinal Plant Knowledge in the Ozarkouachita Highlands. J Ethnobio. 1998;18:249.

33. Lee S, Xiao CJ, Pei SJ. Ethnobotanical survey of medicinal plants at periodic markets of Honghe Prefecture in Yunnan Province. SW China J Ethnopharmacol. 2008;117(2):362-77. doi:10.1016/j.jep.2008.02.001.

34. Yue YY, Zhang WX, Yang R, Zhang QS, Liu ZH. Medicinal Design and operation of an artificial pit for the fermentation of Chinese liquor. J I Brewing. 2007:113(4):374-80. doi:10.1002/j.2050-0416.2007.tb00764.x.

35. Fu ML, Liu J, Chen QH, Liu XJ, He GQ, Chen JC. Determination of ethyl carbamate in Chinese yellow rice wine using high-performance liquid chromatography with fluorescence detection. Int J Food Sci Tech. 2010:45(6):1297-302. doi:10.1111/j.1365-2621.2010.02279.x.

36. Ghorbani A, Langenberger G, Feng L, Sauerborn J. Ethnobotanical study of medicinal plants utilised by Hani ethnicity in Naban river watershed national nature reserve, Yunnan, China. J Ethnopharmacol. 2011;134:651-67. doi:10.1016/j.jep.2011.01.011.

37. Shinwari ZK, Gilani SS. Sustainable harvest of medicinal plants at Bulashbar Nullah, Astore (Northern Pakistan). J Ethnopharmacol. 2003:84(2):289-98.

38. Panyaphu K, Van On T, Sirisa-ard P, Srisa-nga P, ChansaKaow S, Nathakarnkitkul S. Medicinal plants of the Mien (Yao) in Northern Thailand and their potential value in the primary healthcare of postpartum women. J Ethnopharmacol. 2011;135(2):226-37. doi:10.1016/j.jep.2011.03.050.

39. Olsen KM, Purugganan MD. Molecular evidence on the origin and evolution of glutinous rice. Genetics. 2002;162(2):941-50.

40. Sompong R, Siebenhandl-Ehn S, Linsberger-Martin G, Berghofer E. Physicochemical and antioxidative properties of red and black rice varieties from Thailand. China and Sri Lanka Food Chem. 2011;124(1):132-40. doi:10.1016/j.foodchem.2010.05.115.

41. Tananuwong K, Tewaruth W. Extraction and application of antioxidants from black glutinous rice. LWT-Food Sci Technol. 2010;43(3):476-81. doi:10.1016/j.lwt.2009.09.014.
42. Yawadio R, Tanimori S, Morita N. Identification of phenolic compounds isolated from pigmented rices and their aldose reductase inhibitory activities. Food Chem. 2007;101(4):1616-25.

43. Chen PN, Kuo WH, Chiang CL, Chiou HL, Hsieh YS, Chu SC. Black rice anthocyanins inhibit cancer cells invasion via repressions of MMPs and u-PA expression. Chem-Biol Interact. 2006;163(3):218-29. doi:10.1016/j.cbi. 2006.08.003.

44. Lee JC, Kim JD, Hsieh FH, Eun JB. Production of black rice cake using ground black rice and medium-grain brown rice. Int J Food Sci Tech. 2008;43(6):1078-82. doi:10.1111/j.1365-2621.2007.01569.x.

45. Suzuki M, Kimura T, Yamagishi K, Shinmoto H, Yamaki K. Comparison of mineral contents in 8 cultivars of pigmented brown rice. J Jpn Soc Food Sci Tech. 2004:51(58):424-7.

46. Li SM, Long CL, Liu FY, Lee S, Guo Q, Li R, et al. Herbs for medicinal baths among the traditional Yao communities of China. J Ethnopharmacol. 2006;108(1):59-67. doi:10.1016/j.jep.2006.04.014.

47. Long CL, Li R. Ethnobotanical studies on medicinal plants used by the Red-headed Yao People in Jinping, Yunnan Province. China J Ethnopharmacol. 2004;90(2):389-95. doi:10.1016/j.jep.2003.10.021.

48. Giam X, Bradshaw CJA, Tan HTW, Sodhi NS. Future habitat loss and the conservation of plant biodiversity. Biol Conserv. 2010;143(7):1594-602. doi:10.1016/j.biocon.2010.04.019.

49. Giday M, Asfaw Z, Woldu Z. Medicinal plants of Meinit ethnic group of Ethiopia: An ethnobotanical study. J Ethnopharmacol. 2009;124(3):513-21. doi:10.1016/j.jep.2009.05.009.

50. Tolossa K, Debela E, Athanasiadou S, Tolera A, Ganga G, Houdijk JGM. Ethno-medicinal study of plants used for treatment of human and livestock ailments by traditional healers in South Omo. Southern Ethiopia J Ethnobiol Ethnomed. 2013;9:32. doi:10.1186/1746-4269-9-32.

51. Ghorbani A, Langenberger G, Sauerborn J. A comparison of the wild food plant use knowledge of ethnic minorities in Naban River Watershed National Nature Reserve, Yunnan. SW China J Ethnobiol Ethnomed. 2012:8(1):17. doi:10.1186/1746-4269-8-17.

52. Huai HY, Pei SJ. Plants used medicinally by folk healers of the Lahu people from the autonomous county of Jinping Miao, Yao, and Dai in Southwest China. Econ Bot. 2004;58(1):S265-73. doi:10.1663/00130001(2004)58[S265:PUMBFH]2.0.CO;2.

\section{Submit your next manuscript to BioMed Central and take full advantage of:}

- Convenient online submission

- Thorough peer review

- No space constraints or color figure charges

- Immediate publication on acceptance

- Inclusion in PubMed, CAS, Scopus and Google Scholar

- Research which is freely available for redistribution 